\title{
Energetic formulation for rate-independent processes: remarks on discontinuous evolutions with a simple example
}

\author{
Roberto Alessi
}

the date of receipt and acceptance should be inserted later

\begin{abstract}
This work aims to discuss, in the simplest way, some open issues concerning the energetic formulation for the modeling of rate-independent processes by means of a simple one-dimensional example.

Early and unphysical jumps are avoided taking advantage of a time-reparametrization and enforcing an energy imbalance during time-discontinuous transitions. A new energetic selective criterion is then proposed, able to predict the final state after a jump and representing intrinsically the energy contribution of rate-dependent phenomena as inertia and viscosity that may arise during fast discontinuous transitions. An improved version of the energetic formulation is finally proposed, preserving completely the rateindependent setting.

All concepts and results are accompanied by explicative pictures which, hopefully, will help the nonspecialist reader, to whom this paper is addressed, in the understanding of the discussion.
\end{abstract}

Keywords Energetic formulation · rate-independent processes · Variational methods · stability · discontinuities

\section{Introduction}

This work aims to discuss some open issues concerning the energetic formulation for the modeling of rate-independent processes, [43] and references therein, taking advantage of a simple one-dimensional example first introduced by Mielke in [38]. The discussion is deliberately kept to its maximum level of simplicity allowing non-specialist readers to capture primarily the main ideas of this fruitful formulation. The energetic formulation was developed and formalized by mathematicians in the field of Calculus of Variations but mostly applied to describe mechanical problems. Specifically, this theory has led to significant advances under many points of view in the fields of: fracture mechanics, [12, 23, 31, 36]; damage mechanics [21, 51, 50]; plasticity [17, 18, 59, 22, 25]; coupled damage-plasticity modeling [15, 3, 19, 4, 54]; shape-memory alloys modeling $[9,34,2,5]$ and many others.

The ingredients of the energetic formulation are an internal potential energy, a dissipation distance and the power of external actions. In the context of standard generalized materials, the energy potential and the dissipation distance are defined through a potential energy density and a dissipation potential respectively, [24, 37]. The energetic formulation is then characterized by three energetic requirements, an energy balance, a dissipation inequality and a global stability criterion.

The energetic formulation owns considerable advantages compared to classical ones, as, for instance, the following: it offers a rigorous process of analysis starting from minimal assumptions; in the most general setting, it is completely derivative-free admitting discontinuities not only in space but also in time; it allows the use of advanced mathematical tools from Calculus of Variations as Direct Methods for the proof of existence of solutions; it gives a clear meaning to bifurcation and stability concepts

R. Alessi

Dipartimento di Matematica

Università di Roma La Sapienza

Piazzale Aldo Moro 5, 00185 Roma IT

Dipartimento di Ingegneria Strutturale e Geotecnica

Università di Roma La Sapienza

Via Eudossiana 18, 00184 Roma IT

E-mail: roberto.alessi@uniroma1.it 
for both structures and materials; last, but not least, it furnishes a natural and rational way for a numerical implementation since a functional minimization is involved rather than evolution equations, $[11,8,33,36,58,7,6]$ for significant numerical examples in different rate-independent contexts. Classical results automatically descend (for free) in case of smooth evolutions and sufficiently regular functionals as the principle of virtual powers, Karush-Kuhn-Tucker relations, associate flow rules and consistency conditions for internal state variables together with their boundary conditions.

Among all these advantages, some issues need to be highlighted which are discussed in this work. Global stability is a major drawback of the energetic formulation and can be replaced by a "local" stability criterion which is more physical, provided one is able to specify a topology in which neighborhoods are well defined, [37]. Moreover, globally stable solutions descending from non-convex functionals often experience unphysical early and long range jumps, as pointed out by Stefanelli [60] and Roubíček [57], violating the causality principle. In addition Stefanelli noticed in [60] that the conservation of energy at jumps is also questionable. Indeed, at a time discontinuity, rate-dependent phenomena, not taken into account in the energy balance, are likely to occur as, for instance, viscous and inertia effects, which have a strong impact on the response. Another concern is the assessment of the solution sensibility to material or geometrical imperfections as the occurrence of multiple localizations in a perfect damageable bar under traction, [49], but the discussion about this point is postponed to future works.

For sake of completeness, we must mention that other broad classes of solution notions for rateindependent systems exist, [43] for a remarkable overview. In particular, [43, Sect. 1.8] presents a complete list of these solution notions while [43, Fig. 1.8] gives an effective graphical interpretation of their correlation. These solution concepts have been mostly developed and formalized in the mathematical community of Calculus of Variations and their variety reflect the discussion in the engineering community whether inelastic phenomena are energy or stress driven. More specifically, this work has some connections with parametrized solutions, vanishing-viscosity solutions (also called approximable solutions), [18, 13, 30, 32, 16], and balanced viscosity solutions (BV-solutions), [40, 41, 42]. Although limited to a one-dimensional setting, the latter have been carefully characterized and compared with the energetic solutions in [53]. Moreover, BV-solutions can be related, under suitable technical assumptions, to parametrized solutions and can be considered as an extension and generalization of vanishing-viscosity solutions. Compared to energetic solutions, the three aforementioned solutions rely on a differential inclusion and an extended version of the energy balance with an underlying viscosity structure. Nevertheless, we focus here our attention only on energetic solutions, since "full" stability will be taken into account and a true rate-independent setting (without "viscous" like regularizations) discussed.

Specifically, the paper is structured as follows. Sect. 2 is devoted to the introduction of the energetic formulation. First, we set the three energetic ingredients, namely the internal potential energy, the dissipated energy and the power of external actions, that respectively specify how the system stores and dissipates energy as well as how it exchanges energy with the surrounding environment. Then we are able to introduce the governing principles of the evolution, that is the energy balance, the dissipation inequality and different stability criteria: global, local and differentiable. In Sect. 3 we present the one-dimensional rate-independent example, by our knowledge first introduced by Mielke in [38], consisting of a body, initially massless, pulled over a rough surface towards a smoother surface through a spring stretched by a quasi-static loading rate. Here, this example is revisited through a fully parametric analysis which allows to highlight the above mentioned issues. First, global stability is abandoned in favor of a local directional stability condition. Then, the occurrence of early jumps, even with the local stability criterion, is pointed out and a new energetic criterion introduced, which relies on a Lipschitz time-reparametrization and on an energetic imbalance. Jumps are then forced to occur as late as possible, that is only if the evolution reaches a sharp corner of the stability domain. Forcing the evolution to jump as late as possible induces another issue, that is energy conservation at a discontinuous transition leading possibly to a non-existence of an energetically consistent stable state. The conservation of energy can be restored by introducing in the model rate-dependent effects. Concerning the sledge example, this situation is explored by endowing the body with a mass and by replacing the linear spring with a viscous Kelvin-Voigt rheological model. Hence, the rate-dependent jump transition is explicitly investigated obtaining closed form results. Further applications of inertia and viscous regularizations can be found in [55, 56, 1, 29, 54]. Motivated by these results, an improved energetic formulation is proposed in Sect. 4 taking advantage of the time reparametrization and introducing an additional energetic selective criterion, which enclose the one proposed by Stefanelli in [60] and is able to restore energy balance and existence of the response at a jump. Implications of such updated formulation in a multidimensional setting will be discussed in future works.

All concepts and results are accompanied by explicative pictures which, hopefully, will help the nonspecialist reader, to whom this paper is addressed, in the understanding of the discussion. 
Notations The dependence of the generic (vector-valued) variable $\boldsymbol{z}$ on the time evolution parameter $t$ is indicated either by a subscript, e.g. $\boldsymbol{z}_{t}$, or explicitly, e.g. $\boldsymbol{z}(t)$. The dot stands for the time derivative, e.g. $\dot{\boldsymbol{z}}_{t}=\mathrm{d} \boldsymbol{z}_{t} / \mathrm{d} t$. The left- ad right-hand limits $\boldsymbol{z}^{-}\left(t_{j}\right)$ and $\boldsymbol{z}^{+}\left(t_{j}\right)$ are respectively defined as

$$
\boldsymbol{z}^{-}\left(t_{j}\right):=\lim _{t \rightarrow t_{j}^{-}} \boldsymbol{z}(t), \quad \boldsymbol{z}^{+}\left(t_{j}\right):=\lim _{t \rightarrow t_{j}^{+}} \boldsymbol{z}(t)
$$

\section{The energetic formulation}

This section intends to summarize, without referring to all mathematical details, the fundamentals of the energetic formulation able to describe rate-independent evolution problems. The solution of such problems is a time parametrized family of states $\boldsymbol{z}$, called solution process and denoted $\boldsymbol{z}_{t}$, from some time interval $[0, T]$ into a finite- or infinite-dimensional state space $Z$, i.e. $\boldsymbol{z}_{t}:[0, T] \rightarrow Z$. When no source of confusion can occur, the process $\boldsymbol{z}_{t}$ will be also denoted $\boldsymbol{z}(t)$. A typical rate-independent evolution problem consists of an initial state $\boldsymbol{z}_{0} \in Z$ with $\boldsymbol{z}_{0}:=\boldsymbol{z}(0)$ and of course of some time-dependent data, as, for instance in the present mechanical context, prescribed forces or displacements which vary in a quasistatic way. For sake of simplicity, the solution process is hereafter assumed to be piecewise continuous with respect to time. Moreover every continuous part is assumed to be absolutely continuous and Cantor parts, that are taken into account in the general theory, are excluded.

A generic instant where a time-discontinuity of the process occurs is denoted with $t_{j} \in J\left(\boldsymbol{z}_{t}\right)$, being $J\left(\boldsymbol{z}_{t}\right):=\left\{t_{1}, \ldots, t_{n_{\mathrm{j}}}\right\}$ the ordered times jumps set and $n_{\mathrm{j}}$ the number of jumps up to time $t$ of the process $\boldsymbol{z}_{t}$.

The energetic formulation is characterized by three energetic requirements, an energy balance (first law of thermodynamics), a dissipation inequality (second law of thermodynamics) and a global stability criterion. At this stage, it is useful to fix some basic concepts taking a look to Fig. 1, which gives a simplified overview of the features of an expected solution process. The first evidence is that the solution process must always belong to the stable set space $\mathcal{S}$, being consistent with the dissipation inequality and following an energetic isohypse (curve with constant total energy). The occurrence of jumps must not surprise in case of non-convex energetic quantities. Uniqueness is in general not ensured because extended jump regions and bifurcation points with bifurcation paths may arise.

\subsection{Basic energetic quantities}

The energetic formulation is based on three energetic quantities that specify how the system stores and dissipates energy as well as how it exchanges energy with the surrounding environment.

The stored energy, also called the internal potential energy or Helmholtz free energy depending on the context, is a state function given by $\mathcal{E}(\boldsymbol{z})$ with $\mathcal{E}: Z \rightarrow \mathbb{R}$, henceforth assumed suitable smooth and in case non-convex. The conjugate forces $\boldsymbol{\sigma}$ to the state variables $\boldsymbol{z}$ are defined, according to [47], as

$$
\boldsymbol{\sigma}:=\partial_{\boldsymbol{z}} \mathcal{E}(\boldsymbol{z})
$$

In the present context of standard generalized materials, [24], the dissipative behaviour of the system is characterized by a dissipation potential $\varphi(\boldsymbol{z}, \dot{\boldsymbol{z}})$ with $\varphi: T Z \rightarrow[0, \infty]$ and $T Z$ being the tangent bundle of $Z$. This potential is convex, positive, vanishes for null rates and 1-homogeneous with respect to the rates, this last property ensuring the rate independence of the system, [43]. Since the evolution may develop jumps, it is convenient to avoid time derivatives in the formulation. Therefore, consistently with [37], the dissipation distance $d: Z \times Z \rightarrow[0, \infty]$ is introduced based on the Finslerian dissipation metric $\varphi$, as

$$
d\left(\boldsymbol{z}_{0}, \boldsymbol{z}_{1}\right):=\inf \left\{\int_{0}^{1} \varphi(\tilde{\boldsymbol{z}}(s), \dot{\tilde{\boldsymbol{z}}}(s)) \mathrm{d} s: \tilde{\boldsymbol{z}} \in \mathrm{C}^{1}([0,1], Z), \tilde{\boldsymbol{z}}(0)=\boldsymbol{z}_{0}, \tilde{\boldsymbol{z}}(1)=\boldsymbol{z}_{1}\right\}
$$

where the introduction of a minimizing deformation path $\tilde{\boldsymbol{z}}(s)$ always allows to measure the amount of energy dissipated among two states, [48].

Although definition (2) is still under debate in the scientific community and can be simplified in case of a sufficient geometric structure for $Z$, the underlying concept of time stretching (or time reparametrization) will be of great importance in the understanding of subsequent passages. This same idea can be found in the definition of the reduced derivative, [44], or parametrized BV-solutions, [41].

The dissipated energy in the time interval $[0, t]$ is the sum of two contributions, the energy dissipated respectively in absolutely continuous subprocesses $(\cdot \mathrm{c})$ and the at jumps $\left(\cdot{ }_{\mathrm{j}}\right)$,

$$
\mathcal{D}\left(\boldsymbol{z}_{t} ;[0, t]\right)=\mathcal{D}_{\mathrm{c}}\left(\boldsymbol{z}_{t} ;[0, t]\right)+\mathcal{D}_{\mathrm{j}}\left(\boldsymbol{z}_{t} ;[0, t]\right)
$$




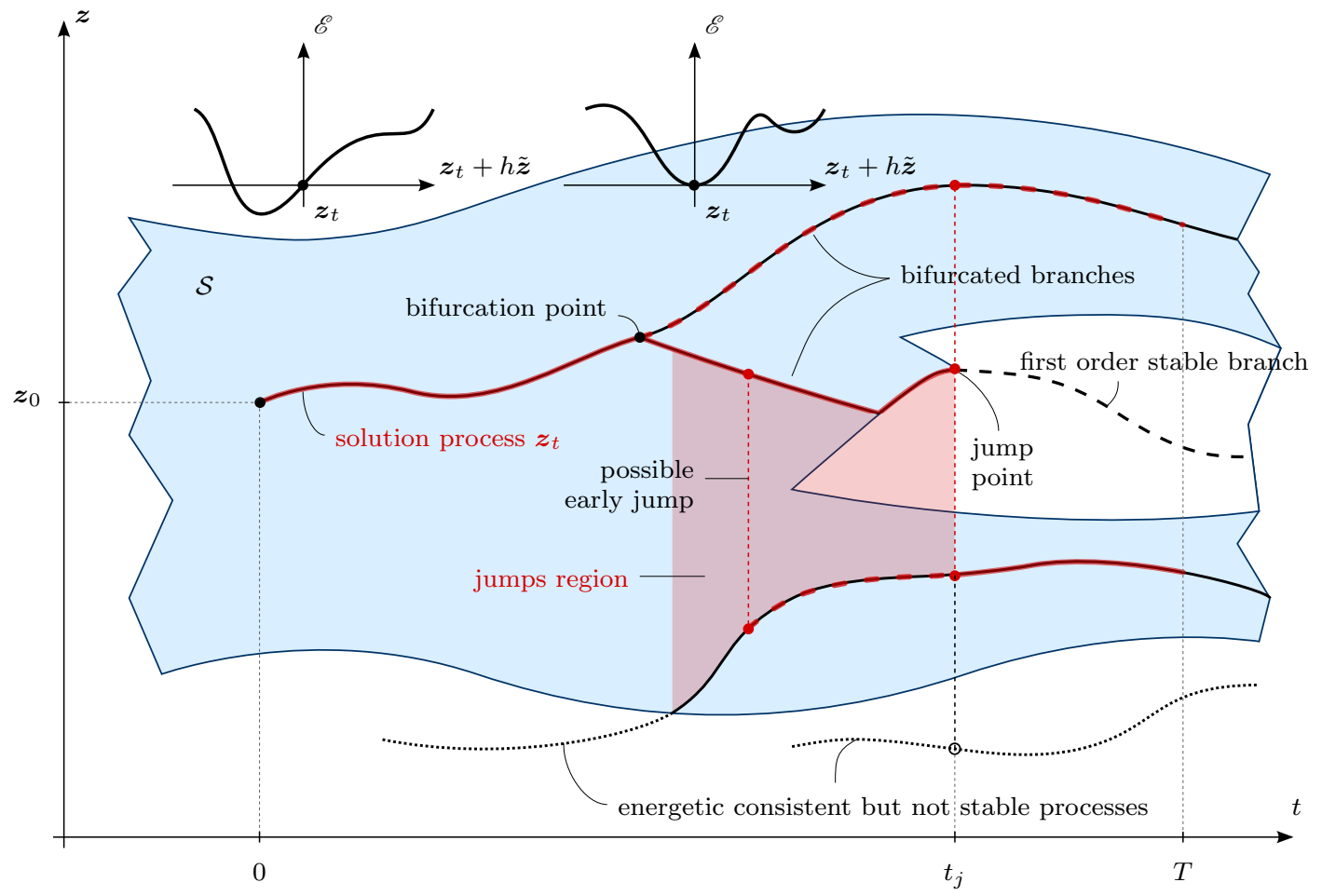

Fig. 1: Graphical interpretation of the energetic formulation principles and relative energetic solutions in a time interval $[0, T]$. The light blue set $\mathcal{S}$ identifies all (locally) stable states while black lines represent evolutions conserving total energy $\mathscr{E}$ (energetic isohypses): continuous, dashed and dotted respectively for stable, first order stable and unstable processes. The realizable processes with the occurrence of possible discontinuities and bifurcations are represented by a red thickened line: continuous for the assumed solution process while dashed for other admissible paths as bifurcated processes or processes reached by a jump

with

$$
\mathcal{D}_{\mathrm{c}}\left(\boldsymbol{z}_{t} ;[0, t]\right)=\int_{0}^{t} \varphi\left(\boldsymbol{z}_{s}, \dot{\boldsymbol{z}}_{s}\right) \mathrm{d} s, \quad \text { and } \quad \mathcal{D}_{\mathrm{j}}\left(\boldsymbol{z}_{t} ;[0, t]\right)=\sum_{t \in J\left(\boldsymbol{z}_{t}\right)} d\left(\boldsymbol{z}_{t}^{-}, \boldsymbol{z}_{t}^{+}\right)
$$

Similarly, the interaction between the system and the surrounding environment is characterized by an external power. In the present context, the contribution to the external power is only due to external forces, $\boldsymbol{f}(t):[0, T] \rightarrow Z^{*}$, with $Z^{*}$ being the dual space of $Z$, and prescribed states, $\overline{\boldsymbol{z}}(t):[0, T] \rightarrow Z$. The external power then reads

$$
\ell\left(t, \dot{\boldsymbol{z}}_{t}\right)=\boldsymbol{f}(t) \cdot \dot{\boldsymbol{z}}_{t}+\boldsymbol{f}_{\mathrm{r}} \cdot \dot{\overline{\boldsymbol{z}}}(t)
$$

with $f_{\mathrm{r}}$ being the reaction forces. Without loss of generality, these external actions are assumed to be smooth (at least continuous) such to comply with the quasi-static setting. Similarly to the dissipation distance, at a fixed time, an external work distance can be then introduced between two states $\boldsymbol{z}_{0}$ and $\boldsymbol{z}_{1}$,

$$
l\left(t ; \boldsymbol{z}_{0}, \boldsymbol{z}_{1}\right):=\int_{\boldsymbol{z}_{0}}^{\boldsymbol{z}_{1}} \ell(t, \boldsymbol{y}) \mathrm{d} \boldsymbol{y}=\boldsymbol{f}(t) \cdot\left(\boldsymbol{z}_{1}-\boldsymbol{z}_{0}\right)
$$

As for the dissipated energy (3), the total energy supplied to the system from instant 0 to $t$ by the surrounding environment can be expressed as

$$
\mathcal{L}\left(\boldsymbol{z}_{t} ;[0, t]\right)=\mathcal{L}_{\mathrm{c}}\left(\boldsymbol{z}_{t} ;[0, t]\right)+\mathcal{L}_{\mathrm{j}}\left(\boldsymbol{z}_{t} ;[0, t]\right)
$$

with

$$
\mathcal{L}_{\mathrm{c}}\left(\boldsymbol{z}_{t} ;[0, t]\right)=\int_{0}^{t} \ell(s, \dot{\boldsymbol{z}}(s)) \mathrm{d} s, \quad \text { and } \quad \mathcal{L}_{\mathrm{j}}\left(\boldsymbol{z}_{t} ;[0, t]\right)=\sum_{t_{j} \in J\left(\boldsymbol{z}_{t}\right)} l\left(t_{j} ; \boldsymbol{z}_{j}^{-}, \boldsymbol{z}_{j}^{+}\right)
$$

and the shorthand notation $\boldsymbol{z}_{j}^{-/+}=\boldsymbol{z}^{-/+}\left(t_{j}\right)$. The quantities $\mathcal{L}_{\mathrm{c}}$ and $\mathcal{L}_{\mathrm{j}}$ represent the external work of conservative actions during absolute continuous subprocesses and at discontinuous transitions respectively. 


\subsection{The Global and Local energetic formulation}

The three energetic principles that characterize the energetic formulation are: an energy balance, a dissipation inequality and a stability criterion. While energy balance and the dissipation inequality are nothing but particular statements of the first and second law of thermodynamics, they are not enough to characterize in general the solution process and further restrictions, given by the stability criterion, are necessary.

A process $\boldsymbol{z}_{t}$ is energetically consistent with the environment if the following energy balance holds:

$$
\mathscr{E}\left(\boldsymbol{z}_{t} ;[0, t]\right):=\mathcal{E}\left(\boldsymbol{z}_{t}\right)-\mathcal{E}\left(\boldsymbol{z}_{0}\right)+\mathcal{D}\left(\boldsymbol{z}_{t} ;[0, t]\right)-\mathcal{L}\left(\boldsymbol{z}_{t} ;[0, t]\right)=0, \quad \forall t \in[0, T]
$$

Besides, we get directly at a jump point that

$$
\mathcal{E}\left(\boldsymbol{z}_{j}^{+}\right)-\mathcal{E}\left(\boldsymbol{z}_{j}^{-}\right)+d\left(\boldsymbol{z}_{j}^{-}, \boldsymbol{z}_{j}^{+}\right)-l\left(t_{j} ; \boldsymbol{z}_{j}^{-}, \boldsymbol{z}_{j}^{+}\right)=0, \quad \forall t_{j} \in J\left(\boldsymbol{z}_{t}\right)
$$

A process $\boldsymbol{z}_{t}$ is said to satisfy the dissipation inequality if, at each $t \in[0, T]$ the rate of energy dissipated at any point is non-negative,

$$
\varphi\left(z_{t}, \cdot\right) \geq 0, \quad \forall t \in[0, T]
$$

which implies $\dot{\mathcal{D}}\left(\boldsymbol{z}_{t} ;[0, t]\right) \geq 0$.

The dissipation inequality (DI) is here ensured by the fact that the dissipation potential is positive, 1-homogeneous and vanishes for null rates.

It is worth noting that constraints on the evolution of some state variables can be prescribed either by letting the dissipation potential attaining an infinite value, [21], or by restricting the state space and its variation as, for instance, in damage mechanics where the scalar damage variable cannot decrease, [51]. Such monotone restriction on the evolution of a state variable is often called irreversibility condition, [43].

Energy balance and positive dissipation are well understood and widely accepted unlike the stability condition. Concerning this last mostly two version exist, the global and local stability condition. In addition, different local stability conditions may be defined based on the topology on $Z$. Hereafter, only the directional and differential versions of the stability condition will be addressed, particular suitable for continuous state variables, $[50,2]$. We suggest the reader to refer to $[39,43]$ for different and more general definitions.

A process $\boldsymbol{z}_{t}$ satisfies the global stability condition if $\forall t \in[0, T]$ and $\forall \tilde{\boldsymbol{z}} \in \widetilde{Z}$

$$
\mathcal{E}\left(\boldsymbol{z}_{t}\right) \leq \mathcal{E}(\tilde{\boldsymbol{z}})+d\left(\boldsymbol{z}_{t}, \tilde{\boldsymbol{z}}\right)-l\left(t ; \boldsymbol{z}_{t}, \tilde{\boldsymbol{z}}\right)
$$

where the admissible state $\widetilde{Z}$ may coincide with $Z$ or, as already pointed out, embed some further explicit irreversibility constraints.

On the other hand a process $\boldsymbol{z}_{t}$ satisfies the directional local stability condition if at any time $t \in[0, T]$ and every $\tilde{\boldsymbol{z}} \in \widetilde{Z}$, with $\widetilde{Z}$ being here the space of admissible test directions, there exists a continuation interval $[0, \bar{h}]$ with $\bar{h} \geq 0$ such that the free energy change induced by any radial continuation process starting from $\boldsymbol{z}_{t}$ does not exceed the corresponding dissipated energy and the external work, namely for $\forall t \in[0, T]$ and $\forall \tilde{\boldsymbol{z}} \in \widetilde{Z}, \exists \bar{h}>0: \forall h \in[0, \bar{h}]$

$$
\mathcal{E}\left(\boldsymbol{z}_{t}\right) \leq \mathcal{E}\left(\boldsymbol{z}_{t}+h \tilde{\boldsymbol{z}}\right)+d\left(\boldsymbol{z}_{t}, \boldsymbol{z}_{t}+h \tilde{\boldsymbol{z}}\right)-l\left(t, \boldsymbol{z}_{t}, \boldsymbol{z}_{t}+h \tilde{\boldsymbol{z}}\right)
$$

It is worth noting that in case of a discrete state-space, instead of (st), a different local stability definition must be given based on a suitable metric and topology on $Z$.

Global stability means that the actual state realizes a global minimum while local stability considers local minima as solution candidates. If $\mathcal{S}_{G}$ and $\mathcal{S}$ represent the sets of all states satisfying respectively the global (ST) and local stability (st) condition, then

$$
\mathcal{S}_{G} \subseteq \mathcal{S}
$$

More specifically one expects $\mathcal{S}_{G}$ to be much smaller than $\mathcal{S}$. A typical case where the two sets coincide is when $\mathcal{E}$ is convex and $\mathcal{D}$ translational invariant.

With sufficient smoothness assumptions, the directional stability condition turns to a differential directional stability condition. Indeed, developing in series the right-hand side (st), one gets the following 
sufficient (and necessary) differential conditions to asses local stability. A process is directionally stable at order $n$ if (and only if) $\forall t \in[0, T] \exists n \geq 1$, with $n \in \mathbb{N}$, is such that

$$
\forall \tilde{\boldsymbol{z}} \in \mathcal{S}_{(n-1)}\left(\boldsymbol{z}_{t}\right), \quad \mathcal{E}^{(n)}\left(\boldsymbol{z}_{t}\right)(\tilde{\boldsymbol{z}})+\mathcal{D}_{0}^{(n)}\left(\boldsymbol{z}_{t}\right)(\tilde{\boldsymbol{z}})-\mathcal{L}_{0}^{(n)}\left(\boldsymbol{z}_{t}\right)(\tilde{\boldsymbol{z}})>(\geq) 0
$$

where, $\mathcal{D}_{0}\left(\boldsymbol{z}_{t}\right):=\mathcal{D}\left(\boldsymbol{z}_{t} ;[0, t]\right), \mathcal{L}_{0}\left(\boldsymbol{z}_{t}\right):=\mathcal{L}\left(\boldsymbol{z}_{t} ;[0, t]\right), \mathcal{X}^{(n)}\left(\boldsymbol{z}_{t}\right)(\tilde{\boldsymbol{z}})$ is the $n$-th Gateaux derivative of $\mathcal{X}$ at $\boldsymbol{z}_{t}$ in direction $\tilde{\boldsymbol{z}}$ and, moreover, if $n>1$,

$$
\forall \bar{k} \in[0, n-1] \text { and } \forall \tilde{\boldsymbol{z}} \in \bigcap_{k=0}^{\bar{k}} \mathcal{S}_{(k)}\left(\boldsymbol{z}_{t}\right), \quad \mathcal{E}^{(k)}\left(\boldsymbol{z}_{t}\right)(\tilde{\boldsymbol{z}})+\mathcal{D}_{0}^{(k)}\left(\boldsymbol{z}_{t}\right)(\tilde{\boldsymbol{z}})-\mathcal{L}_{0}^{(k)}\left(\boldsymbol{z}_{t}\right)(\tilde{\boldsymbol{z}}) \geq 0
$$

where the subsets of directions to be tested at each order are given by

$$
\mathcal{S}_{(k)}\left(\boldsymbol{z}_{t}\right):= \begin{cases}\widetilde{Z}, & k=0 \\ \left\{\tilde{\boldsymbol{z}} \in \widetilde{Z} \mid \mathcal{E}^{(k)}\left(\boldsymbol{z}_{t}\right)(\tilde{\boldsymbol{z}})+\mathcal{D}_{0}^{(k)}\left(\boldsymbol{z}_{t}\right)(\tilde{\boldsymbol{z}})-\mathcal{L}_{0}^{(k)}\left(\boldsymbol{z}_{t}\right)(\tilde{\boldsymbol{z}})=0\right\}, & k \geq 0\end{cases}
$$

The differential version of stability has been fruitful applied in several contexts for the study of structural or material stability. The first order differential stability condition is essentially equal to the virtual work principle or to a stationary condition.

In the following, processes satisfying $(\mathrm{EB})$ and $(\mathrm{ST}) /(\mathrm{st})$ will be denoted global/local energetic solutions or energetic consistent globally/locally stable solutions.

\section{Some issues occurring in a simple one-dimensional example}

In this section we face the issue concerning discontinuous evolutions by means of a simple one-degree of freedom rate-independent system, by our knowledge first introduced by Mielke in [38]. Nevertheless, therein, the example is only briefly analyzed with numerical constitutive values which led to partial results. Here, the analysis is extended to be fully parametric and novel outcomes are discussed. Most of the implications of this analysis apply also to multidimensional settings but will be discussed in future works.

The problem, schematically represented in Fig. 2, consists of a body, at this stage massless, pulled over a rough surface towards a smoother surface through a spring stretched with a quasi-static loading rate. The state of the system is described by a single state variable $p \in Z \equiv \mathbb{R}$, representing contemporary the position of the body and of one spring end. The associated test direction space is $\widetilde{Z} \equiv \mathbb{R}$. A quasi-static prescribed displacement $u(t)$ is instead applied to the other spring end.

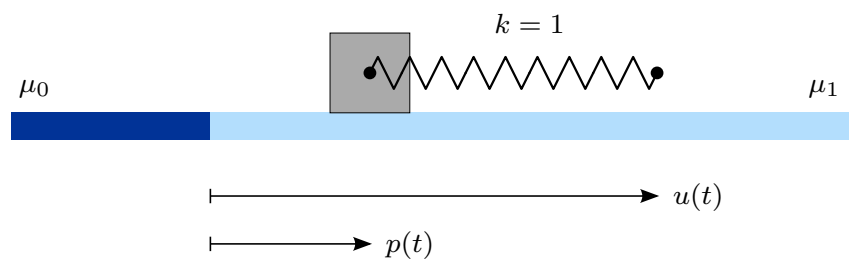

Fig. 2: Schematic representation of the examined rate-independent system (sledge example), [38]

The stored energy of this system is totally enclosed in the linear elastic spring of unitary stiffness $(k=1)$, and therefore given by

$$
\mathcal{E}(u(t)-p)=\frac{1}{2}(u(t)-p)^{2}
$$

The stress, derived from the stored energy, (1), simply reads

$$
\sigma=(u-p)
$$

Since no external forces are applied but only a prescribed displacement, the external power reduces to

$$
\ell(t, \dot{p})=\ell(t)=f_{\mathrm{r}} \dot{u}(t)
$$

where $f_{\mathrm{r}}$ is the reaction force of the spring at the pulled extremity and equals, accordingly to the energy balance (EB), the stress (14). 
The only source of dissipation in the system is, at this stage, friction modeled by the dissipation potential

$$
\varphi(p, \dot{p})=\mu(p)|\dot{p}|, \quad \text { with } \quad \mu(p)= \begin{cases}\mu_{0}, & \text { if } p \leq 0 \\ \mu_{1}, & \text { if } p>0\end{cases}
$$

where $\mu_{0}$ and $\mu_{1}$ represent the friction coefficients of the rough and smooth surfaces respectively such that $\mu_{0}>\mu_{1}$.

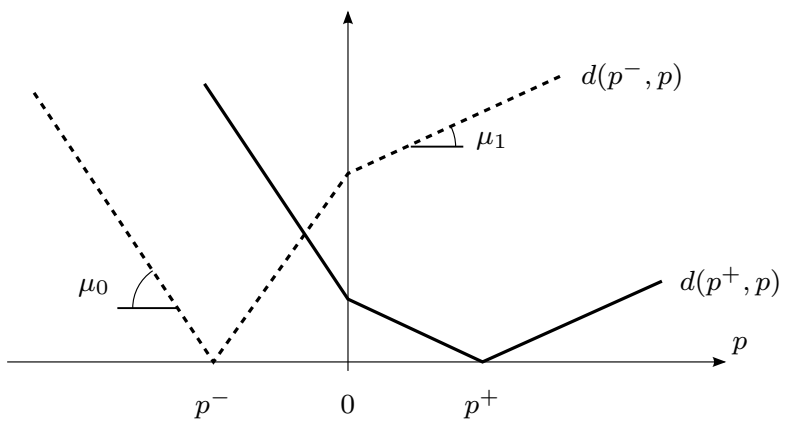

Fig. 3: Graph of the dissipation distance $d\left(p^{ \pm}, \cdot\right)$ for $p^{-}<0$ and $p^{+}>0$ with slopes belonging to the set $\left\{ \pm \mu_{0}, \pm \mu_{1}\right\}$. The functions $d\left(p^{+}, p\right)$ and $d\left(p^{-}, p\right)$ are respectively convex and non-convex with respect to $p$

Since the dissipation distance is not convex for $p \leq 0$, Fig. 3, different results between global stability and local stability are expected as well as the occurrence of jumps in the evolution.

Without loss of generality, hereafter we assume as prescribed displacement law $u(t)=t$, as initial instant $t_{0}=-\mu_{0}$ and as initial state $p_{0}=-\mu_{0}$, such that $\left(t_{0}, p_{0}\right)$ corresponds to a stable state.

\subsection{Energetic solutions}

The solution of the evolution problem is here built and discussed in several steps:

(S1) First, the global/local stable states are determined for any instant $t$, that is the states that the system can virtually attain;

(S2) Then, candidate energetic solutions are highlighted by enforcing energy balance. Globally stable solutions are found to be bad candidates and therefore, for the remainder of this presentation, excluded.

(S3) Finally, all candidate processes, possibly discontinuous, are discussed with respect to other solution notions. An enriched version of the energy balance (EB) is proposed, taking advantage of the time reparametrization concept, which avoids early jumps.

\subsubsection{The global and local stability domain $\mathcal{S}_{G}$ and $\mathcal{S}$}

The global stability condition (ST) allow us to define the global stability domain $\mathcal{S}_{G} \in \mathbb{R} \times \mathbb{R}$. In particular, for the present example,

$$
p \in \mathcal{S}_{G} \Leftrightarrow \forall q \in \mathbb{R}, \quad \frac{1}{2} p^{2}-t p \leq \frac{1}{2} q^{2}-t q+ \begin{cases}\mu_{0}|q-p|, & \text { if } p \leq 0, q \leq 0 \\ \mu_{1}|q-p|, & \text { if } p>0, q>0 \\ \mu_{0}|q|+\mu_{1}|p|, & \text { if } p>0, q \leq 0 \\ \mu_{1}|q|+\mu_{0}|p|, & \text { if } p \leq 0, q>0\end{cases}
$$

The set $\mathcal{S}_{G}$ of admissible global stable states is represented in Fig. 4a.

On the other hand, the local stability condition (st) defines the local stability domain $\mathcal{S} \in \mathbb{R} \times \mathbb{R}$. In particular, by straightforward calculations, we get

$$
p \in \mathcal{S} \Leftrightarrow \begin{cases}|t-p| \leq \mu_{0}, & \text { if } p<0 \\ |t-p| \leq \mu_{1}, & \text { if } p \geq 0\end{cases}
$$

It is worth noting that the set $\mathcal{S}$ of admissible local stable states, represented in Fig. $4 \mathrm{~b}$, has a sharp corner at $(t, p)=\left(\mu_{0}, 0\right)$ and that $\mathcal{S}_{G} \subset \mathcal{S}$, according to (10). 


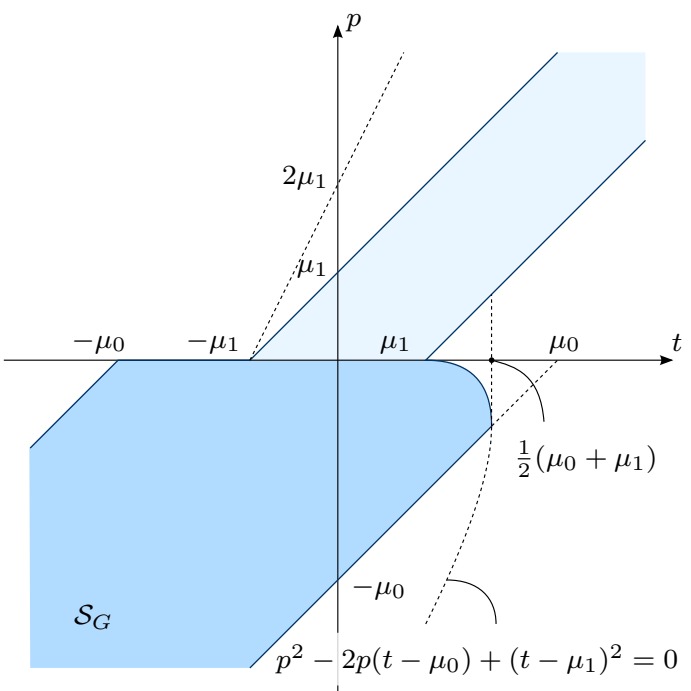

(a)

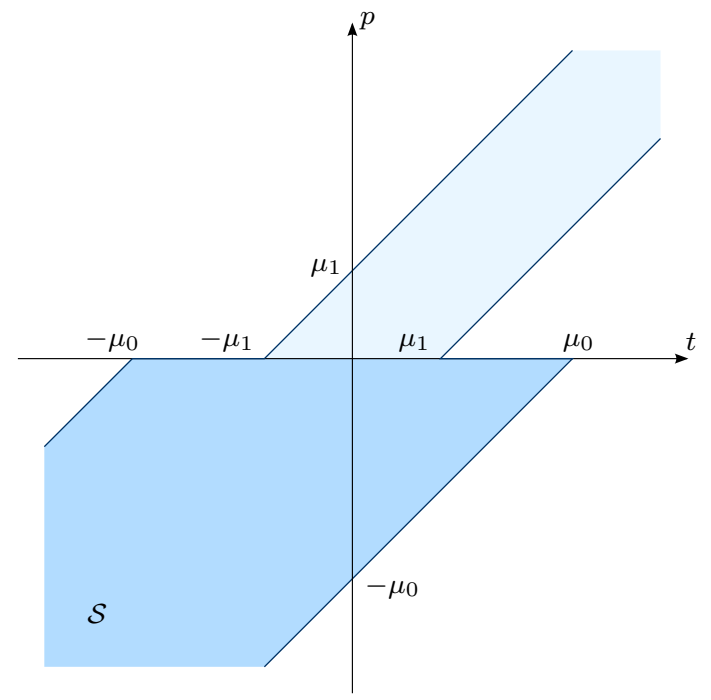

(b)

Fig. 4: Global (a) and local (b) stability domains

\subsubsection{The global and local energetic candidate solutions}

In the previous section the global and local stable states, compatible with the external loading, have been established, Fig. 4. The aim of this section is to determine all candidate energetic processes $p_{i}(t)$, according to step (S2), by exploring the implications of (EB).

For what concerns global energetic solutions, it turns out, by straightforward calculations, that the admissible process is unique, namely

$$
p_{t}= \begin{cases}-\mu_{0}, & \text { for }-\mu_{0} \leq t \leq 0 \\ t-\mu_{0}, & \text { for } 0<t \leq\left(\mu_{0}+\mu_{1}\right) / 2 \\ t-\mu_{1}, & \text { for } t>\left(\mu_{0}+\mu_{1}\right) / 2\end{cases}
$$

depicted with a red curve in Fig. 5.

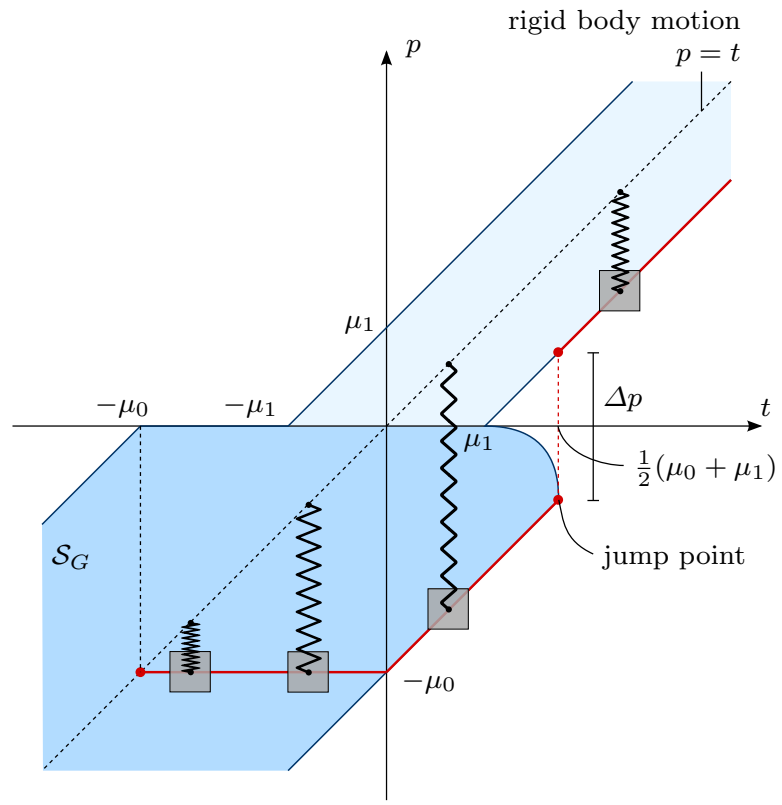

Fig. 5: Global energetic solution with the occurrence of a too-early jump 
It is then worth noting that at $t=\left(\mu_{0}+\mu_{1}\right) / 2=: t_{\mathrm{j}}$ a jump in the evolution must occur, of amplitude $\Delta p=p^{+}\left(t_{\mathrm{j}}\right)-p^{-}\left(t_{\mathrm{j}}\right)=\left(\mu_{0}-\mu_{1}\right)$ with $p^{+}\left(t_{\mathrm{j}}\right)=\left(\mu_{0}-\mu_{1}\right) / 2$ and $p^{-}\left(t_{\mathrm{j}}\right)=-\left(\mu_{0}-\mu_{1}\right) / 2$. It is then clear that such evolution is "nonphysical" since it violates the principle of causality. Indeed the jump occurs well before the friction coefficient changes its value. Such behaviour has been already explained and highlighted in [38]. Therefore global energetic solutions are hereafter excluded although they have some merits as, for instance, in the modeling of discrete problems, where it is difficult to define a proper locality notion, [21], or in the modeling of the initiation of a brittle fracture, [14, 35] and [27] for a technical discussion.

For what concerns local energetic solutions, it turns out that a continuous family of possible processes exist. More specifically two situations have to be distinguished, for reasons that will be clear in a moment, namely the case where $\mu_{0} \leq 3 \mu_{1}$, corresponding to a first family of processes denoted $p_{t, n}$, and the case where $\mu_{0}>3 \mu_{1}$ corresponding to a second family of processes $q_{t, n}$, with $n \in[0,1]$ being a continuous parameter describing all single solutions.

Then, for $\mu_{0} \leq 3 \mu_{1}$, the $p_{t, n}$ local solution processes are

$$
p_{t, n}=\left\{\begin{array}{ll}
-\mu_{0}, & \text { for }-\mu_{0} \leq t \leq 0 \\
t-\mu_{0}, & \text { for } 0<t<t_{\mathrm{j}_{n}} \\
p_{n}^{+}, & \text {for } t_{\mathrm{j}_{n}} \leq t \leq p_{n}^{+}+\mu_{1} \\
t-\mu_{1}, & \text { for } t>p_{n}^{+}+\mu_{1}
\end{array}, \quad n \in[0,1]\right.
$$

with

$$
t_{\mathrm{j}_{n}}=n \mu_{0}+(1-n)\left(\mu_{0}+\mu_{1}\right) / 2
$$

and

$$
p_{n}^{+}=\frac{1}{2}\left(\mu_{0}-\mu_{1}\right)((1+n)+2 \sqrt{n})
$$

being respectively the instant where a jump occurs and the state attained immediately after the jump. This family of solution processes is represented in Fig. 6a.

For $\mu_{0}>3 \mu_{1}$, the $q_{t, n}$ local solution processes are

$$
q_{t, n}=\left\{\begin{array}{ll}
-\mu_{0}, & \text { for }-\mu_{0} \leq t \leq 0 \\
t-\mu_{0}, & \text { for } 0<t<s_{\mathrm{j}_{n}} \\
q_{n}^{+}, & \text {for } s_{\mathrm{j}_{n}} \leq t \leq q_{n}^{+}+\mu_{1} \\
t-\mu_{1}, & \text { for } t>q_{n}^{+}+\mu_{1}
\end{array}, \quad \forall n \in[0,1]\right.
$$

with

$$
s_{\mathrm{j}_{n}}=n \bar{t}+(1-n)\left(\mu_{0}+\mu_{1}\right) / 2, \quad \bar{t}:=\frac{3 \mu_{1}^{2}+\mu_{0}^{2}}{2\left(\mu_{0}-\mu_{1}\right)}
$$

and $q_{n}^{+}=p_{n}^{+}$defined accordingly to (21). This other family of solution processes is represented in Fig. $6 \mathrm{~b}$.

In addition, another locally stable admissible process exists, at least up to $t=\mu_{0}$, which is

$$
p_{t}^{*}=\left\{\begin{array}{cl}
-\mu_{0}, & \text { for }-\mu_{0} \leq t \leq 0 \\
t-\mu_{0}, & \text { for } 0<t<\mu_{0} \\
? & \text { for } t \geq \mu_{0}
\end{array}\right.
$$

and has, at this stage, no continuation for $t>\mu_{0}$ in sense of local energetic solutions. Indeed there does not exist any stable state $p^{+} \in \mathcal{S}$ at $t=\mu_{0}$ able to satisfy the energy balance jump relation (9) with $p^{-}=0$. Nevertheless, up to $t=\mu_{0}$, the process $(24)$ is exactly the unique expected physical response, Fig. $6 \mathrm{c}$ !

It is then clear that local energetic solutions allow for (if $\mu_{0} \leq 3 \mu_{1}$ ) or are obliged (if $\mu_{0}>3 \mu_{1}$ ) to realize an early nonphysical jump. In addition, this last case embeds the possibility to jump at $t=0$ but without conserving energy. To understand better these facts, allowing to formulate an improved energetic formulation, it is useful to look to the energy plots at five different stages of the response, highlighted with circled numbers in Fig. $6 \mathrm{~b}$, and for the most general case, $\mu_{0}>3 \mu_{1}$.

In these pictures $p_{t}$ is the current state while $\mathscr{E}=\mathscr{E}\left((s-1) p_{t}+s \tilde{p} ;[0,1]\right)$ represents the energy quantity (EB) with respect to any varied state $\tilde{p}$ along a virtual process parametrised by $s$ and constructed as a linear combination of $p_{t}$ and $\tilde{p}$. Moreover the energy plot $\mathscr{E}$ is superimposed to the local stable region $\mathcal{S}$ highlighting admissible multiple solutions. When $p_{t} \leq-\mu_{0}+\mu_{1}$ the solution is unique as we can observe from Fig. 7-(1). Indeed, no other states different from $p_{t}$ and such that $\mathscr{E}=0$ exist. Moreover the locally stable region $\mathcal{S}$ is a connected set. 


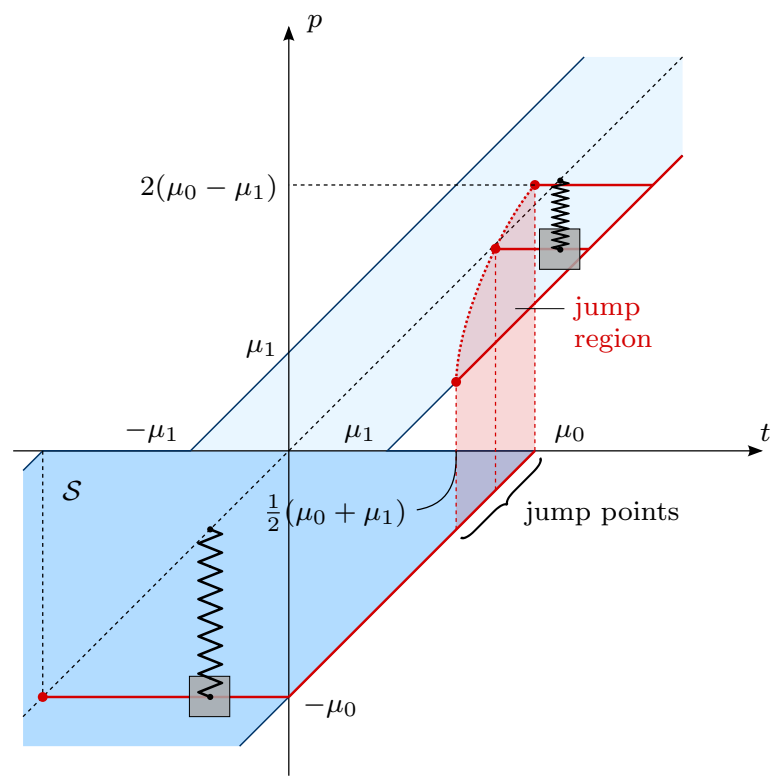

(a) $\mu_{0} \leq 3 \mu_{1}$

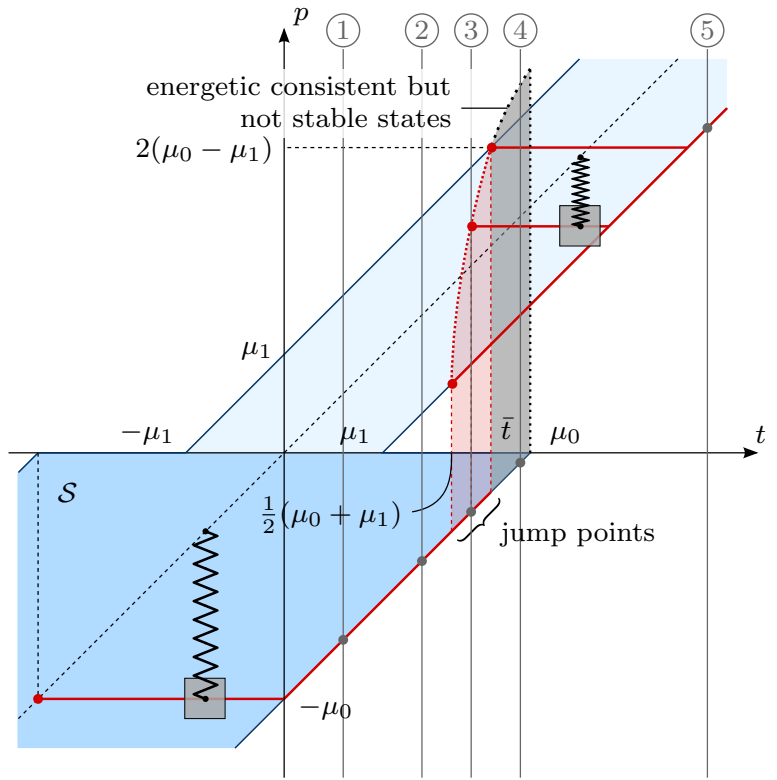

(b) $\mu_{0}>3 \mu_{1}$

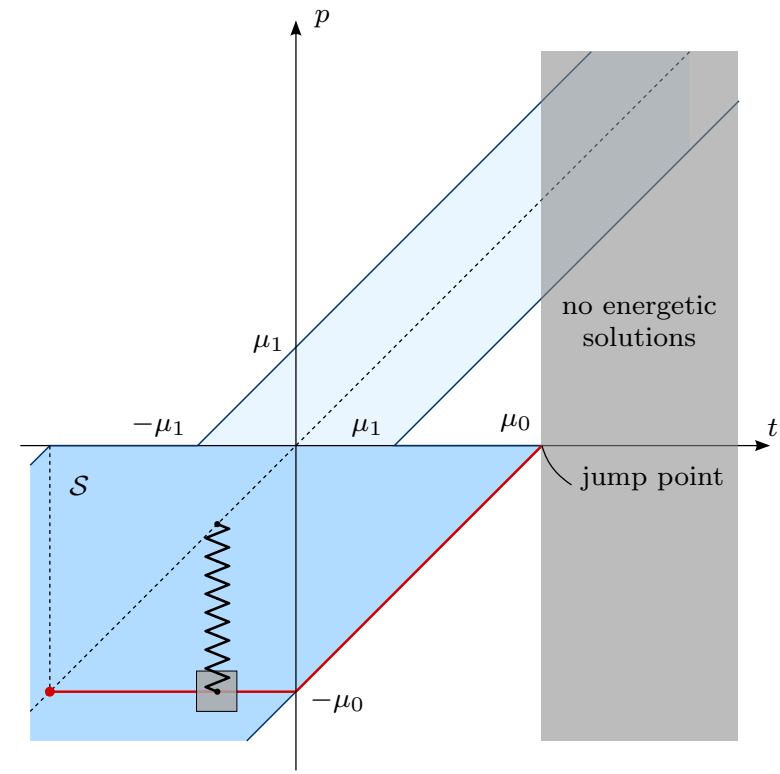

(c) $\mu_{0}>3 \mu_{1}$

Fig. 6: Families of local energetic solution processes for different constitutive parameters with occurrence of discontinuities: $\mu_{0} \leq 3 \mu_{1}$, (a), and $\mu_{0}>3 \mu_{1}$, (b)-(c). Admissible and non-admissible jump regions are highlighted respectively with a red and gray transparent areas. Red paths represent the continuous solution part (continuous lines), the jump trajectories (dashed lines) and the final jump states (dotted lines)

As long as $\mu_{1}-\mu_{0}<p_{t}<\left(\mu_{1}-\mu_{0}\right) / 2$ the response continues to be unique except for the limit case $p_{t}=\left(\mu_{1}-\mu_{0}\right) / 2$ where another possible solution appears, $\tilde{p}=\left(\mu_{0}-\mu_{1}\right) / 2$, since, for this state, $\mathscr{E}=0$ (Fig. 7-2)). When $-\left(\mu_{0}-\mu_{1}\right) / 2 \leq p_{t} \leq \bar{t}-\mu_{0}$ two energetic consistent solutions appear with $\mathscr{E}=0$. However the smallest one has to be excluded since it lies outside $\mathcal{S}$, Fig. 7-3), while the other one is admissible and identified by (21). But for $\bar{t}-\mu_{0} \leq p_{t} \leq 0$, also the former possible solution becomes inadmissible since it falls back outside $\mathcal{S}$, Fig. 7-(4). Nevertheless at this stage $p_{t}$ continues to be an admissible solution. The limit case $p_{t}=0$, where no admissible jumps may occur and where $p_{t}$ is on the sharp boundary corner of the stable set $\mathcal{S}$, not allowing further continuous evolutions, is depicted further on in Fig. 14b. In case the evolution has experienced an admissible jump, Fig. 7-5 shows the energy 

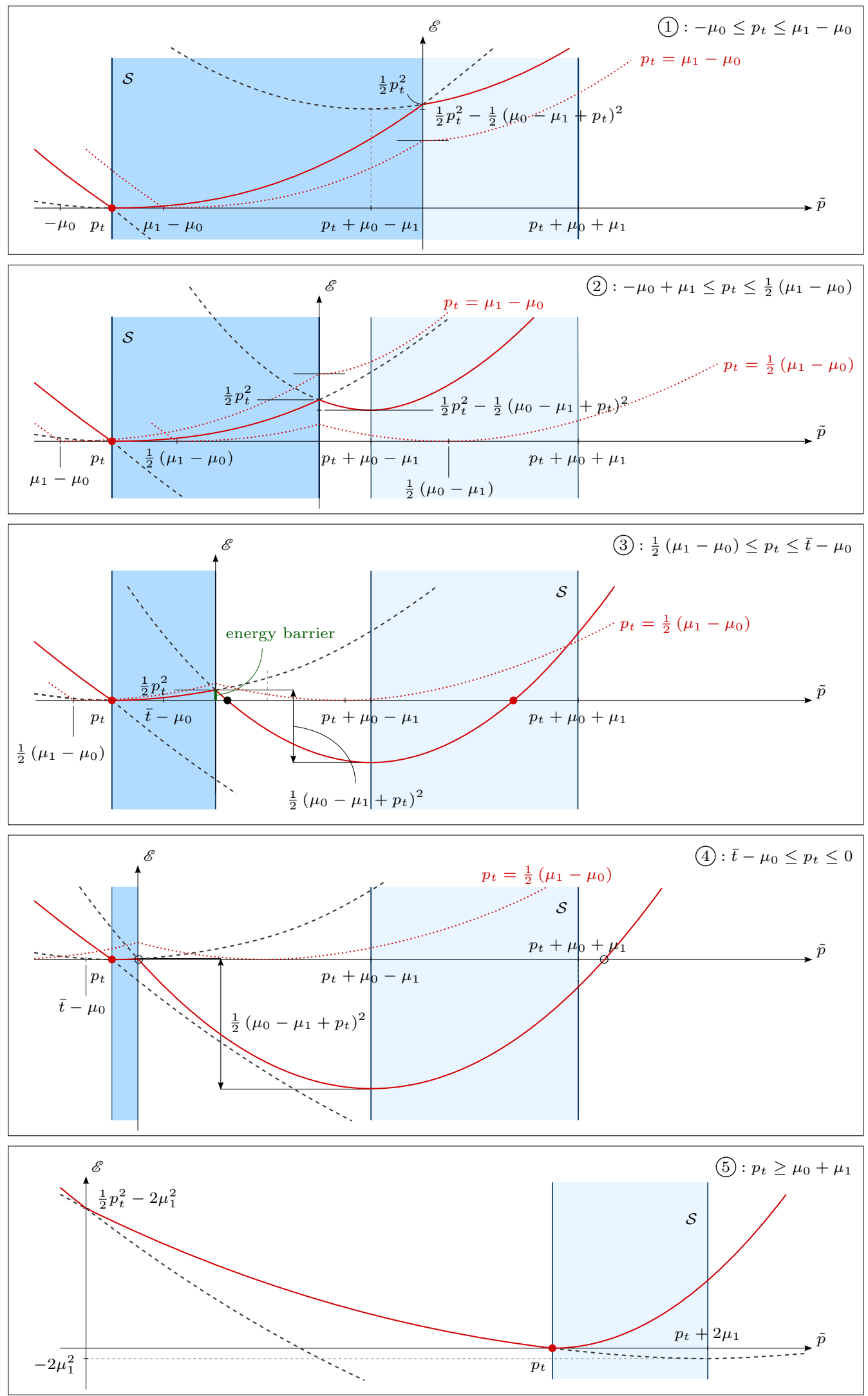

Fig. 7: Energy landscapes $\mathscr{E}$ (continuous red line) at different states $p_{t}, \odot$ in Fig. 6b. Dashed black lines and dotted red lines correspond respectively to construction curves and limit energy profiles. The light blue regions represent the stability domain 
profile for states during the stationary evolution $p_{t} \geq \mu_{0}+\mu_{1}$ corresponding to the continuously sliding body on the smoother surface.

By far, the analysis carried out here is more general than the one in [38] where the choice of the friction coefficients were only numerical, such that $\mu_{0}=3 \mu_{1}$, and where no multiple solutions were highlighted for the locally stable process.

\subsubsection{Are early jumps possible? An energy barrier argument}

Motivated by the previous section, it is clear that early jumps must be avoided since they violate the causality principle. An heuristic principle, which overcomes this incongruity, could be the following:

The solution process, satisfying (EB) and (st), is as long as possible continuous

Statement (25) will be motivated rigorously by means of an energy barrier argument after the following preliminary definitions and remarks, concerning continuous evolutions. Indeed (25) has different implications and several scenarios are worth being explored.

A process $\boldsymbol{z}(t)$ is said to be left-/right-continuous at an instant $t$ if

$$
\lim _{\delta t \rightarrow 0^{-} /+} \boldsymbol{z}(t+\delta t)=\boldsymbol{z}(t)
$$

A process $\boldsymbol{z}_{t}$ is said to be continuous at an instant $t$ if it is both left- and right-continuous at the same instant $t$.

Let us now assume that at a generic instant $t^{*}$ there exists a continuous local energetic solution $\boldsymbol{z}(t)$ in the time-interval $\left(t_{0}, t^{*}\right)$ and that in a successive time-interval $\left(t^{*}, t_{1}\right)$ several, for sake of simplicity a finite number $n$, of non-intersecting continuous stable solution candidates $\boldsymbol{z}_{i}(t)$ exist, with $i \in[1, \ldots, n]$. Moreover let us assume that at least $\boldsymbol{z}(t)$ or any $\boldsymbol{z}_{i}(t)$ are left- or right-continuous at $t^{*}$. We are looking now for a continuous solution process $\boldsymbol{z}^{*}(t)$ in the time interval $t \in\left(t_{0}, t_{1}\right)$. Then, several scenarios are possible:

- If there exists only one candidate solution, say $\boldsymbol{z}_{1}$ with $n \geq 1$, such that

$$
\lim _{\delta t \rightarrow 0^{+}} \boldsymbol{z}_{1}\left(t^{*}+\delta t\right)=\lim _{\delta t \rightarrow 0^{-}} \boldsymbol{z}\left(t^{*}+\delta t\right)=\overline{\boldsymbol{z}}
$$

then the process $z^{*}$ is unique, continuous in the time interval $\left(t_{0}, t_{1}\right)$ and given by

$$
\boldsymbol{z}^{*}(t)= \begin{cases}\boldsymbol{z}(t), & t \in\left(t_{0}, t^{*}\right) \\ \overline{\boldsymbol{z}}, & t=t^{*} \\ z_{1}(t), & s \in\left(t^{*}, t_{1}\right)\end{cases}
$$

This situation corresponds to the inner parts of continuous red lines in Figures 5 and 6 .

- If there exist $n>1$ candidate solutions such that $m$ of them, denoted $\boldsymbol{z}_{j}$ with $1<m \leq n$, are such that

$$
\lim _{\delta t \rightarrow 0^{+}} \boldsymbol{z}_{j}\left(t^{*}+\delta t\right)=\lim _{\delta t \rightarrow 0^{-}} \boldsymbol{z}\left(t^{*}+\delta t\right)=: \overline{\boldsymbol{z}}, \quad j \in\{1, \ldots, m\}
$$

then the solution $\boldsymbol{z}^{*}$ in the time interval $\left(t_{0}, t_{1}\right)$ is not unique and the evolution is allowed to follow different continuous branches

$$
\boldsymbol{z}_{j}^{*}(t)=\left\{\begin{array}{ll}
\boldsymbol{z}(t), & t \in\left(t_{0}, t^{*}\right) \\
\overline{\boldsymbol{z}}, & t=t^{*} \\
\boldsymbol{z}_{j}(t), & t \in\left(t^{*}, t_{1}\right)
\end{array}, \quad \text { with } j \in\{1, \ldots, m\}\right.
$$

The state $\overline{\boldsymbol{z}}$ is said to be a bifurcation point, the corresponding instant $t^{*}$ a bifurcation instant and $\boldsymbol{z}_{j}$ bifurcated branches. The existence of multiple equi-probable solutions is rather common in mechanics. Classical examples of both structural and material bifurcation analyses can be found in the context of elastic buckling, where, for instance, the lateral deflection direction of a straight, hinged, elastic and axially critically loaded column is indeterminate, [10], or in the context of damage mechanics, where, for instance, the homogeneous evolution may bifurcate toward a localized solution, [51]. In these situations, material and geometric imperfections have a key role for the definition of the actual response. 
- If all $n \geq 1$ candidate solutions are such that

$$
\lim _{\delta t \rightarrow 0^{+}} \boldsymbol{z}_{i}\left(t^{*}+\delta t\right) \neq \lim _{\delta t \rightarrow 0^{-}} \boldsymbol{z}\left(t^{*}+\delta t\right)=: \hat{\boldsymbol{z}}, \quad \forall i \in\{1, \ldots, n\}
$$

then the process must jump realising a discontinuous evolution. The state $\hat{\boldsymbol{z}}$ is said jump point and the corresponding instant $t^{*}$, jump instant. Fig. 5 highlights the occurrence of a single jump point at $t=\left(\mu_{0}+\mu_{1}\right) / 2$, while Fig. 6 gives an entire time interval where a jump can be expected. Clearly, a sufficient condition for the occurrence of a discontinuity in the evolution is the loss of stability, meaning that the evolution reaches a sharp boundary corner of the stable set $\mathcal{S}$ as highlighted, for instance, in Fig. 1 at the occurrence of the jump point, or for the sledge example at $\left(t, p_{t}\right)=\left(\mu_{0}, 0\right)$.

It may also happen that at a jump instant, no other local energetic solutions exists as in Fig. 6c at $(t, p)=\left(\mu_{0}, 0\right)$. Indeed, at this instant, the energy balance (EB) is violated for any final jump state which falls in the stability domain $\mathcal{S}$.

Moreover, also in the case where an energetic consistent jump occurs according to (9), it is questionable whether the system truly reaches the predicted state since in the fast discontinuous transitions other ratedependent phenomena may become relevant such as inertia or viscosity. The issue of energy conservation at jump points as well as the continuation of the evolution are key points of this work and are faced in the next section (Sect. 3.2).

The attention is now focused on the following question: Why the system should not jump before no longer continuous stable and energetic consistent continuations evolutions exist anymore? The answer we propose is based on the concept of time reparametrization (formally a Lipschitz parametrization of the extended graph, $[20,40,53,46,45])$ of the discontinuous evolution, by now limited to the discontinuity itself in the sense that follows. Let us first assume the occurrence of a jump at instant $t$ in the evolution from state $\boldsymbol{z}_{t}^{-}$to $\boldsymbol{z}_{t}^{+}$. Since in the real world nothing can disappear from one state and instantaneously appear in a completely different state, it is reasonable to assume a continuous transition, regardless how fast, from $\boldsymbol{z}_{t}^{-}$ and $\boldsymbol{z}_{t}^{+}$. This transition can be then parametrized by another evolution parameter, say $s \in[0,1]$, and a "fictitious" process $\hat{\boldsymbol{z}} \in \mathrm{C}([0,1] ; Z)$ such that $\hat{\boldsymbol{z}}(s=0)=\boldsymbol{z}_{t}^{-}$and $\hat{\boldsymbol{z}}(s=1)=\boldsymbol{z}_{t}^{+}$, which can be interpreted as the process of a time rescaling in the fast transition. As already pointed out, this time-rescaling idea is not new and can be also found in the definition of the reduced derivative, [44], of the dissipation distance (2), [37], and as a keystone in the concept of $\varepsilon$-stability proposed by Larsen [26].

Then, along the unknown fast transition process $\hat{\boldsymbol{z}}_{s}$ in the stretched reparametrized time-interval $[0,1]$, an energy imbalance deduced from $(\mathrm{EB})$ is expected to hold since other rate-dependent phenomena, possibly dissipative, are expected to occur such as inertia or viscous effects

$$
\mathcal{E}\left(\hat{\boldsymbol{z}}_{s}\right)-\mathcal{E}\left(\boldsymbol{z}_{t}^{-}\right)+d\left(\boldsymbol{z}_{t}^{-}, \hat{\boldsymbol{z}}_{s}\right)-l\left(t, \boldsymbol{z}_{t}^{-} ; \hat{\boldsymbol{z}}_{s}\right) \leq 0, \quad \forall s \in[0,1]
$$

This condition is quite different from the one proposed by Larsen [26, 27], which instead relies on the $\varepsilon$-slide concept. Indeed, while condition (EI-jump) simply doesn't allow the energy to increase more than its initial value at the jump point during the discontinuous transition, the energy inequality proposed by Larsen constrains the energy to be for the most part decreasing since only increments of amount less then $\varepsilon \ll 1$ are allowed through the entire discontinuous transition.

If (EI-jump) must be valid along the entire jump interval, then it must also hold at an instant as close as possible to $s=0$ such that a linearization of $\hat{\boldsymbol{z}}_{s}$ is possible for all admissible evolution directions. Then, there must exist at least a direction $\tilde{\boldsymbol{z}}$ and an arbitrary small $\bar{h}>0$ such that

$$
\mathcal{E}\left(\boldsymbol{z}_{t}^{-}+h \tilde{\boldsymbol{z}}\right)-\mathcal{E}\left(\boldsymbol{z}_{t}^{-}\right)+d\left(\boldsymbol{z}_{t}^{-}, \boldsymbol{z}_{t}^{-}+h \tilde{\boldsymbol{z}}\right)-l\left(t_{j} ; \boldsymbol{z}_{t}^{-}, \boldsymbol{z}_{t}^{-}+h \tilde{\boldsymbol{z}}\right) \leq 0, \quad \forall h \in[0, \bar{h}]
$$

But in case such direction $\tilde{\boldsymbol{z}}$ exists, condition (ei-jump) would violate the local stability condition (st) except for the case where an equality holds. That is, a jump in the evolution can only occur if the actual state along a continuous evolution becomes unstable. Early jumps are then avoided because of this energy barrier argument, as it happens in Fig. 7-3) for the sledge example, where the energy barrier is explicitly highlighted.

Another existing principle, which claims to avoid early jumps, is the integrated maximum-dissipation principle introduced by Roubíček in [57] and [43, Def. 3.3.8], which seems to be not only ineffective for the sledge example in avoiding early jumps, because it is valid only before the dissipation phenomenon is activated (in other words, before reaching the yield limit), but also needless if adopting the above energy barrier argument. Despite this, it must be credited for the insight given to the relation between the maximal dissipation principle with the local stability condition and for its effectiveness in other cases.

For the above arguments and at this stage, we can state that the energy balance (EB) appears inadequate for a proper description of rate-independent phenomena with non-convex functionals. Therefore it seems reasonable to replace (EB) with a new energetic criterion. For this purpose it is convenient to 
represent $\boldsymbol{z}_{t}$ by a (Lipschitz) parametrization, first suggested in [20], of the form $s \mapsto(\tilde{t}(s), \tilde{\boldsymbol{z}}(s))$ of the extended graph with $\tilde{t}^{\prime}(s) \geq 0,\left\|\tilde{\boldsymbol{z}}^{\prime}(s)\right\| \leq 1$ and normalize the parametrization such that $s \in[0, S]$ with $\tilde{t}(0)=0$ and $\tilde{t}(S)=T$. Without loss of generality and for sake of simplicity we assume $\tilde{t}^{\prime}(s)=1$ if $\boldsymbol{z}(\tilde{t}(s))$ is continuous while $\tilde{t}^{\prime}(s)=0$ at a jump point, Fig. 8 .

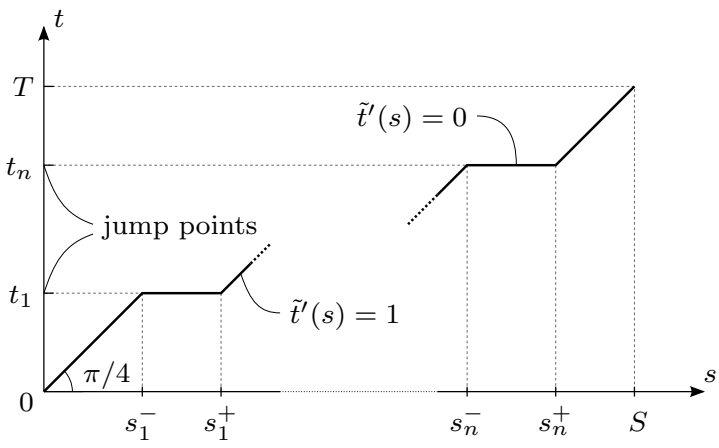

Fig. 8: Time reparametrization function $s \mapsto \tilde{t}(s)$ with $n$ being the number of jump instants, $t_{j}$ with $j \in[1, n]$, of the response

Then, the new proposed energetic criterion states that the solution process $\boldsymbol{z}_{t}$ must satisfy the following condition: $\forall t \in[0, T], \forall s_{0} \in S_{t}^{*}$ and $\forall s_{t} \in S_{t}$,

$$
\mathscr{E}\left(\tilde{\boldsymbol{z}}_{s} ;\left[s_{0}, s\right]\right):=\mathcal{E}\left(\tilde{\boldsymbol{z}}_{s}\right)-\mathcal{E}\left(\tilde{\boldsymbol{z}}_{0}\right)+\mathcal{D}\left(\tilde{\boldsymbol{z}}_{s} ;\left[s_{0}, s\right]\right)-\mathcal{L}\left(\tilde{\boldsymbol{z}}_{s} ;\left[s_{0}, s\right]\right) \leq 0, \quad \forall s \in\left[s_{0}, s_{t}\right]
$$

with $S_{t}^{*}:=\left\{s \in[0, S]: \tilde{t}(s) \leq t\right.$ and $\left.\tilde{t}^{\prime}(s)=1\right\}$ and $S_{t}:=\{s \in[0, S]: \tilde{t}(s)=t\}$.

The energy imbalance (EI), together with (st), is equivalent to the Karush-Kuhn-Tucker like system

$$
\forall s_{t} \in S_{t}, \quad\left\{\begin{array}{l}
\mathscr{E}\left(\tilde{\boldsymbol{z}}_{s},\left[s_{t}^{-}, s\right]\right) \leq 0 \\
\tilde{t}^{\prime}(s)=\{0,1\} \quad, \quad \forall s \in\left[s_{t}^{-}, s_{t}\right] \\
\mathscr{E}\left(\tilde{\boldsymbol{z}}_{s},\left[s_{t}^{-}, s\right]\right) \tilde{t}^{\prime}(s)=0
\end{array}\right.
$$

where

$$
s_{t}^{-}= \begin{cases}s_{j}^{-}, & \text {if } s_{t} \in\left[s_{j}^{-}, s_{j}^{+}\right] \\ s_{j}^{+}, & \text {if } s_{t} \in\left[s_{j}^{+}, s_{j+1}^{-}\right]\end{cases}
$$

Roughly speaking, (32) states that during an absolutely continuous subprocess, the total energy is conserved while at a jump it cannot increase but eventually only decrease.

Condition (EI) is now able to guarantee that the evolution of the sledge example continuously reaches the state $(t, p)=\left(\mu_{0}, 0\right)$ avoiding nonphysical early jumps. Nevertheless, the determinacy of the final state at the jump still remains an issue because at $t=\mu_{0}$ all $p^{+} \in\left[\left(\mu_{0}-\mu_{1}\right), \min \left\{\mu_{0}+\mu_{1}, 2\left(\mu_{0}-\mu_{1}\right)\right\}\right]$ satisfy (EI) and (st).

\subsection{The rate-dependent solution at a jump}

Motivated by the previous remarks and conclusions, this section is devoted to a deeper investigation of the evolution during a jump. In particular, the attention is driven toward the final state $\boldsymbol{z}^{+}$of a jump of a fast rate-dependent transition starting from $\boldsymbol{z}^{-}$. First, the rate-dependent transition $p(s)$ of the sledge example is explicitly solved by comparing viscous, inertia and both coupled effects. Based on such analyses, an additional selective energetic criterion is then proposed by revisiting (9). Concerning the sledge example, this will be able to account also for cases with $3 \mu_{1}<\mu_{0}$, where the local energetic solutions are misleading.

The rate-dependent transition of the sledge example at the jump state $(t, p)=\left(\mu_{0}, 0\right)$ is now investigated by considering in addition inertia and viscous effects simply by assuming the body endowed with a mass $m \geq 0$ and by replacing the linear spring with a Kelvin-Voigt model (linear spring and linear dashpot in parallel) with viscous coefficient $\eta \geq 0$ and same stiffness $k=1$ of the rate-independent setting. 
The governing equation of such continuous time-system, [61], which can be either derived from the Biot equation, [47], or directly deduced from Fig. 9, is

$$
m \ddot{p}_{t}+\eta\left(\dot{p}_{t}-\dot{u}(t)\right)+\mu\left(p_{t}\right) \operatorname{sgn}\left(\dot{p}_{t}\right)-\left(u(t)-p_{t}\right)=0
$$

Due to the quasi-static external loading rate, inertia and viscous effects play a role only at a jump instant, corresponding to $t=\mu_{0}$, such that (33) can be restricted only to the fast rate-dependent jump transition, with time parameter $s \in[0, \infty]$, namely

$$
m \ddot{p}_{s}+\eta \dot{p}_{s}+\mu_{1} \operatorname{sgn}\left(\dot{p}_{s}\right)-\left(\mu_{0}-p_{s}\right)=0
$$

with initial conditions

$$
p(0)=0 \quad \text { and } \quad \dot{p}(0)=0
$$

and where $\mu(p)=\mu_{1}$, since the motion will occur only on the smooth surface. This system then can be regarded as a viscous-friction damped oscillator.

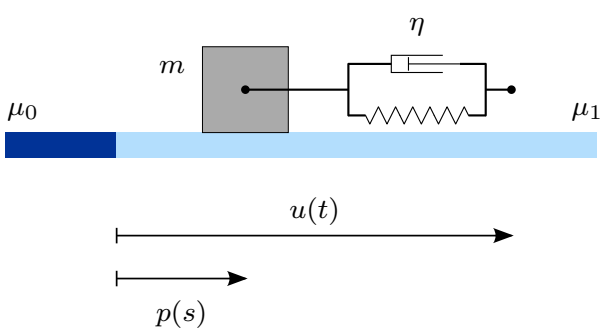

(a)

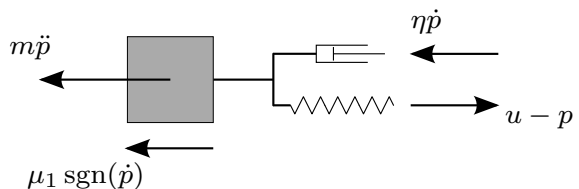

(b)

Fig. 9: Rate dependent model (a) and the corresponding free body diagram (b) for $\dot{p}>0$ and $\ddot{p}>0$

Hereafter the response of such system is investigated considering first only viscous effects $(m=0)$, then only inertia effects $(\eta=0)$ and finally both coupled effects.

\subsubsection{The solution with viscosity}

By considering only viscous effects, that is $\eta \neq 0$ and $m=0$, the governing equation (34) reduces to

$$
\eta \dot{p}_{s}+\mu_{1} \operatorname{sgn}\left(\dot{p}_{s}\right)-\left(\mu_{0}-p_{s}\right)=0
$$

The solution of (36) with boundary conditions (35) then simply reads

$$
p(s)=\left(\mu_{0}-\mu_{1}\right)\left(1-e^{-s / \eta}\right)
$$

which tends asymptotically to

$$
p^{+}:=\lim _{s \rightarrow \infty} p(s)=\mu_{0}-\mu_{1}
$$

as shown in Fig. 10. It is worth noting that during such evolution $\operatorname{sgn}\left(\dot{p}_{s}\right)>0, \forall s>0$.

Regardless of the viscous coefficient $\eta$, which affects only the transition rate but not the final asymptotic solution state, the viscous solution jumps to the first stable state. The dissipation due to viscosity in the time-interval $[0,+\infty]$ is given by

$$
\mathcal{D}_{\text {vis }}=\int_{0}^{+\infty} \eta \dot{p}_{s}^{2} \mathrm{~d} s=\frac{1}{2}\left(\mu_{0}-\mu_{1}\right)^{2}
$$

Both the final state (38) and the dissipated energy (39) coincide with the solution of the vanishingviscosity approach. Moreover it is worth underlying that (39) is exactly the energy amount required to restore energy balance. Indeed, the jump condition (9) returns to be satisfied once the dissipation $\mathcal{D}_{\text {vis }}$ is added to its left-hand side. 


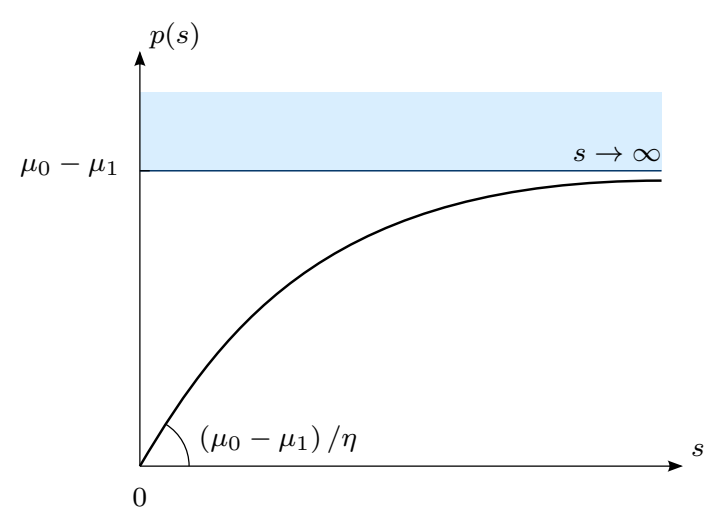

Fig. 10: The viscous solution $p(s)$, (37), during the jump transition

\subsubsection{The solution with inertia}

By considering only inertia effects, that is $\eta=0$ and $m \neq 0$, the governing equation (34) reduces to

$$
m \ddot{p}_{s}+\mu_{1} \operatorname{sgn}\left(\dot{p}_{s}\right)-\left(\mu_{0}-p_{s}\right)=0
$$

Three different cases are worth to be investigated separately, since they will help for a better understanding of the response. These are

(K1) $\mu_{1}=0$, corresponding to an undamped spring-mass system (simple harmonic oscillator);

(K2) $\mu_{0} \leq 3 \mu_{1}$, corresponding to a strongly damped oscillator where the system is expected to converge to a stable state without oscillations;

(K3) $0<3 \mu_{1}<\mu_{0}$, corresponding to a weakly damped oscillator where the system is expected to converge to a stable state with more than one half-oscillation.

In case (K1), the solution will never converge to a stable state with vanishing rate because of the absence of dissipating phenomena while for cases (K2) and (K3) the solution is expected to converge to a fixed state in a finite time but strongly depending on the constitutive parameters $\mu_{0}$ and $\mu_{1}$.

More specifically, the solution for case (K1) is straightforward given by

$$
p(s)=\mu_{0}\left(1-\cos \left(\omega_{\mathrm{n}} s\right)\right)
$$

with $\omega_{\mathrm{n}}:=1 / \sqrt{m}$ being the undamped natural frequency. The solution (41) corresponds to a periodic sinusoidal oscillation of period $2 \pi / \omega_{\mathrm{n}}$, amplitude $2 \mu_{0}$ and mean value $p=\mu_{0}$, Fig. 11a.

On the contrary, the solution for case (K2) corresponds to a single half-oscillation

$$
p(s)=\left(\mu_{0}-\mu_{1}\right)\left(1-\cos \left(\omega_{\mathrm{n}} s\right)\right), \quad s \leq \pi / \omega_{\mathrm{n}}
$$

where the new stable state is reached in a finite time interval of duration $\pi / \omega_{\mathrm{n}}$ with final state

$$
p^{+}:=p\left(\pi / \omega_{\mathrm{n}}\right)=2\left(\mu_{0}-\mu_{1}\right)
$$

as shown in Fig. 11b. It is worth noting that by comparing (21) and (43) with $m=1$, for $\mu_{0} \leq 3 \mu_{1}$, the local energetic solution is correctly described by the solution with inertia but not by the viscous model. Specifically, the final jump state of the strongly damped oscillator corresponds to the farthest stable state consistent with the energy imbalance (EI) which, at the final state, becomes an equality as opposed to the viscous solution (38).

In case (K3), more than one half-oscillation occur. Indeed, the first fixed state after the start of motion coincides with (43) which is located outside the stable set $\mathcal{S}$. Hence, an inversion of motion occurs (overloaded spring compared to friction resistance) giving rise to oscillations of decreasing amplitude until a fixed state, located inside the stable domain, is reached. A single half-oscillation $p_{k}(s)$ is characterized by its initial fixed state $\bar{p}_{k-1}$ and its consecutive fixed state $\bar{p}_{k}$. For the analytic construction of the exact solution, forward $(\mathrm{Fw})$ and reverse $(\mathrm{Rv})$ oscillations must be discriminated in order to deal with the friction force in (34) which depends on $\operatorname{sgn}(\dot{p})$. The system will not evolve any more at the first occurrence of a fixed state belonging to the set $\mathcal{S}$ of locally stable states, namely

$$
\left\{\begin{array}{l}
\left|\bar{p}_{k}-\mu_{0}\right|>\mu_{1}, \quad \forall k \in\{1, \ldots, K-1\} \\
\left|\bar{p}_{k}-\mu_{0}\right| \leq \mu_{1}, \quad k=K
\end{array}\right.
$$


where $K \geq 2$ is assumed to be the minimum number of oscillations needed to reach the fixed state and where the explicit solutions for forward (odd $k$ ) and reverse (even $k$ ) oscillations are

$$
p_{k}(s)= \begin{cases}\left(\mu_{0}-\mu_{1}\right)+\left(\bar{p}_{k-1}-\left(\mu_{0}-\mu_{1}\right)\right) \cos \left(\omega_{\mathrm{n}} s\right), & \text { odd } k \\ \left(\mu_{0}+\mu_{1}\right)+\left(\bar{p}_{k-1}-\left(\mu_{0}+\mu_{1}\right)\right) \cos \left(\omega_{\mathrm{n}} s\right), & \text { even } k\end{cases}
$$

with each final state given by

$$
\bar{p}_{k}= \begin{cases}-\bar{p}_{k-1}+2\left(\mu_{0}-\mu_{1}\right), & \text { odd } k \\ -\bar{p}_{k-1}+2\left(\mu_{0}+\mu_{1}\right), & \text { even } k\end{cases}
$$

By inferring expressions (49) one obtains that the fixed stable state is

$$
p^{+}:=\sum_{k=1}^{K}\left(\left(1-(-1)^{k}\right)\left(\mu_{0}-\mu_{1}\right)+\left(1-(-1)^{k-1}\right) k \mu_{1}\right)
$$

The number of oscillations $K$ can be found by replacing (47) in place of $\bar{p}_{k}$ in (44). An example of a possible weakly damped evolution is represented in Fig. 11c. The total amount of dissipated energy during the transition evolution is due only to friction and reads

$$
\mathcal{D}_{\text {in }}=\left(2 K\left(\mu_{0}-\mu_{1}\right)-\sum_{k=1}^{K} 4(k-1) \mu_{1}\right) \mu_{1}
$$

significantly higher than (2). Moreover, as for the strongly damped evolution, the final state strongly depends on the constitutive parameters and does not coincide with the viscous solution. Nevertheless the final fixed state $p^{+}$is very sensible to the constitutive parameters, as noticeable from (47), and can attain, for instance, any stable state by a continuous variation of $\mu_{1}$, Fig. 12a. Besides, the mass should be of no concern, at least for this one-dimensional model, since the final state is mass-independent and the mass affects only the convergence rate toward the final fixed state. The key point here is that the transition from $p^{-}$to $p^{+}$is not straight due to inertia effects.

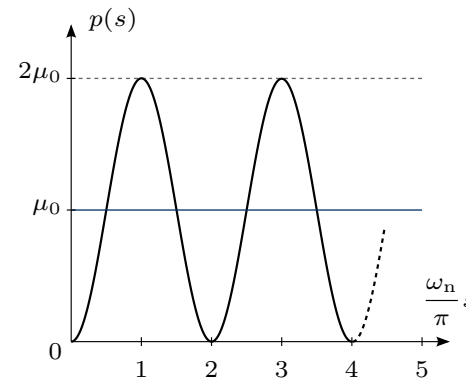

(a)

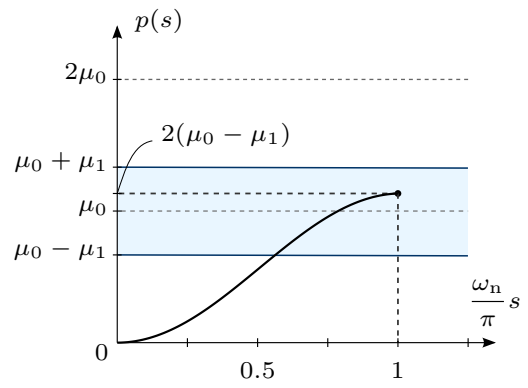

(b)

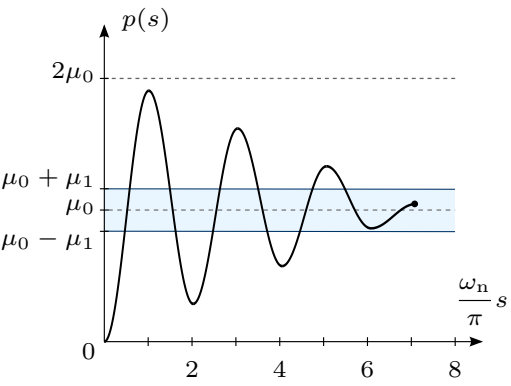

(c)

Fig. 11: The solutions with inertia $p(s)$, (37), during the jump transition for different values of $\mu_{1}$ : (a) $\mu_{1}=0$, (b) $3 \mu_{1} \geq \mu_{0}$ and (c) $0<3 \mu_{1}<\mu_{0}$

\subsubsection{The solution with viscosity and inertia}

In this section, both inertia and viscosity are contemporarily taken into account in (34). As for the solution with only inertia, the system is expected to converge after some $K \geq 1$ oscillations to a fixed stable state. Forward $(\mathrm{Fw})$ and reverse $(\mathrm{Rv})$ oscillations, starting from an arbitrary initial state $\bar{p}_{k-1}$, read

$$
p_{k}(s)= \begin{cases}\left(\mu_{0}-\mu_{1}\right)+\left(\bar{p}_{k-1}-\mu_{0}+\mu_{1}\right)\left(\cos \left(\omega_{\mathrm{d}} s\right)+\frac{\xi}{\sqrt{1-\xi^{2}}} \sin \left(\omega_{\mathrm{d}} s\right)\right) e^{-\xi \omega_{\mathrm{n}} s}, & \text { odd } k \\ \left(\mu_{0}+\mu_{1}\right)+\left(\bar{p}_{k-1}-\mu_{0}-\mu_{1}\right)\left(\cos \left(\omega_{\mathrm{d}} s\right)+\frac{\xi}{\sqrt{1-\xi^{2}}} \sin \left(\omega_{\mathrm{d}} s\right)\right) e^{-\xi \omega_{\mathrm{n}} s}, & \text { even } k\end{cases}
$$




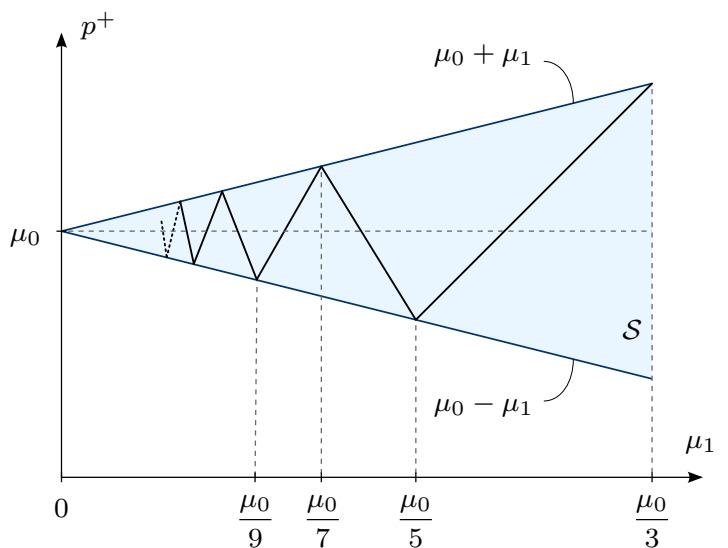

(a)

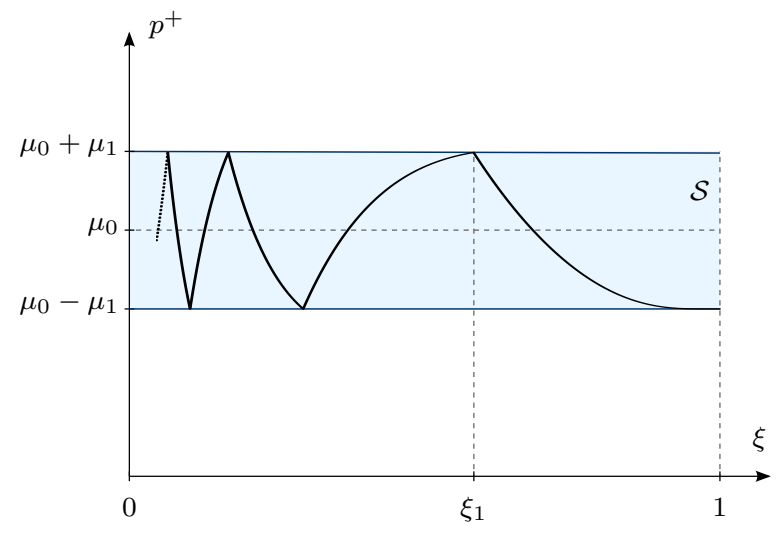

(b)

Fig. 12: Dependence of the final state $p^{+}$at a jump transition with respect to the friction coefficient $\mu_{1}$ for the model (K3), (a), and with respect to the damping ratio $\xi$ for the model (V3), (b)

where $k \in\{1, \ldots, K\}, \xi:=\eta \omega_{\mathrm{n}} / 2$ is the damping ratio and $\omega_{\mathrm{d}}:=\omega_{\mathrm{n}} \sqrt{1-\xi^{2}}$ is the frequency of free oscillations termed damped natural frequency, [61], with $\omega_{\mathrm{n}}$ defined in (41). In particular, after the initiation of motion, the system achieves its first fixed state at finite or infinite time $s$, depending whether the damping ratio $\xi$ is less or greater then one.

More specifically, the following cases are worth to be separately investigated:

(V1) $\xi>1$, corresponding to an overdamped oscillator;

(V2) $\xi=1$, corresponding to a critically damped oscillator;

(V3) $\xi<1$, corresponding to an underdamped oscillator.

Case (V1) corresponds to an evolution for which only one oscillation occurs, $K=1$ and

$$
p(s)=\left(\mu_{0}-\mu_{1}\right)+\left(\mu_{1}-\mu_{0}\right)\left(\cos \left(\omega_{\mathrm{d}} s\right)+\frac{\omega_{\mathrm{n}} \xi}{\omega_{\mathrm{d}}} \sin \left(\omega_{\mathrm{d}} s\right)\right) e^{-\omega_{\mathrm{n}} \xi s}
$$

The fixed state is asymptotically reached for an infinite transition time interval and reads

$$
p^{+}=\lim _{s \rightarrow \infty} p(s)=\mu_{0}-\mu_{1}
$$

which equals the viscous solution (38).

Similarly, also case (V2) corresponds to a solution for which only one oscillation occurs, $K=1$. By straightforward calculations we easily get

$$
p(s)=\left(\mu_{0}-\mu_{1}\right)\left(1-\left(1+\omega_{\mathrm{n}} s\right) e^{-\omega_{\mathrm{n}} s}\right)
$$

The final fixed state coincides with (52) and is reached for an infinite time interval as well. Comparing the viscous solution (37) with the critically damped viscous-solution with inertia (52) one gets that the former tends slower to the coinciding final state $p^{+}$with respect to the latter due to the additional linear function $\omega_{\mathrm{n}} s$.

On the other hand, case (V3) corresponds to a solution for which more oscillations occur, $K>1$. The solution of a single oscillation is determined with (49), while the final state $\bar{p}_{k}$, attained for a finite time interval, is given by

$$
\bar{p}_{k}= \begin{cases}\left(\mu_{0}-\mu_{1}\right)-\left(\bar{p}_{k-1}-\mu_{0}+\mu_{1}\right) e^{-\frac{\pi \xi}{\sqrt{1-\xi^{2}}}}, & \text { odd } k \\ \left(\mu_{0}+\mu_{1}\right)-\left(\bar{p}_{k-1}-\mu_{0}-\mu_{1}\right) e^{-\frac{\pi \xi}{\sqrt{1-\xi^{2}}}}, & \text { even } k\end{cases}
$$

The farthermost stable fixed state attained with one oscillation, $K=1$, is given by solving

$$
\bar{p}_{1}-\mu_{0}=\mu_{1}, \quad \rightarrow \quad \xi_{1}:=\frac{\log \left(\frac{\mu_{0}-\mu_{1}}{2 \mu_{1}}\right)}{\sqrt{\pi^{2}+\log \left(\frac{\mu_{0}-\mu_{1}}{2 \mu_{1}}\right)^{2}}}
$$




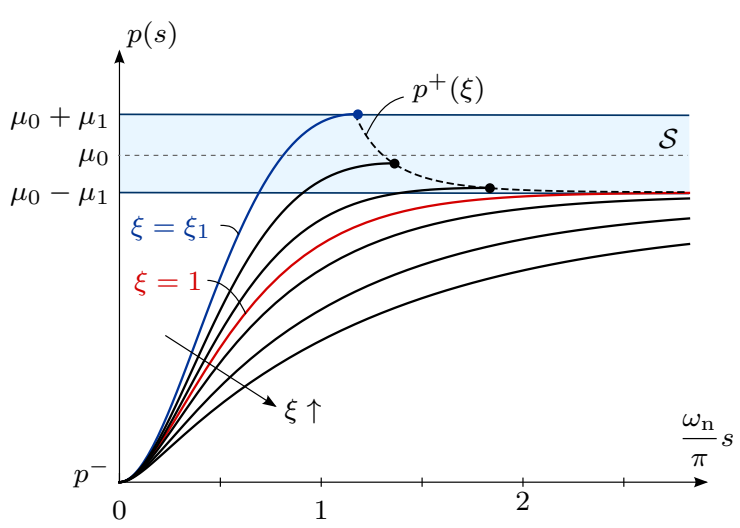

(a)

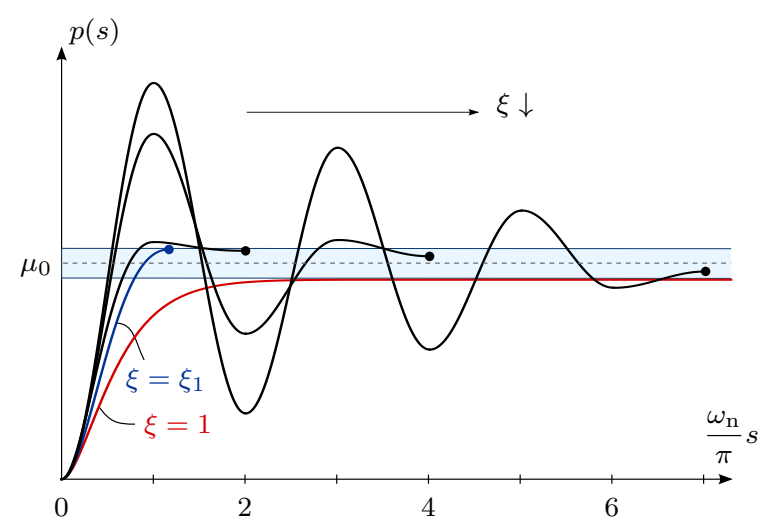

(b)

Fig. 13: The inertia-viscous solution $p(s)$ during the jump transition for different values of $\xi$ : (a) $\xi \geq \xi_{1}$, (50) and (52), (b) $\xi \leq \xi_{1}$, (49)

such that, for continuity, all admissible stable states at a jump can be reached letting $\xi$ varying in the interval

$$
\xi_{1} \leq \xi<1
$$

and provided that $\mu_{0} \geq 3 \mu_{1}$, as highlighted in Fig. 13a. For $\xi \leq \xi_{1}$ all stable states can be achieved as well but with multiple oscillations, Fig. $13 \mathrm{~b}$.

While the final stable state of the viscous solution, (38), and of the solution with inertia, (43) and (47), do not depend respectively on the viscous coefficient $\eta$ and on the mass $m$, the final stable state of the viscous-inertia solution strongly does through $\xi$, (53), at least for $\xi<1$, Fig. 12b.

\subsection{Conclusions}

In this simple example we have shown that the final state after a jump strongly depends on the competition between viscous and inertia effects. The energy balance (9) may then be violated either because other dissipative phenomena are triggered or because the transition from $p^{-}$to $p^{+}$is not straight. Indeed, while the viscous solution always tends to the closest stable state, (38), solutions with inertia do not. As soon as both inertia and viscous effects are taken into account, different solutions may occur depending on their competition through the value of $\xi$, Tab. 1. In Figures $12 \mathrm{a}$ and 12b, we have shown at the jump how the tuning of the friction coefficient $\mu_{1}$ and the viscous parameter $\xi$ can lead to complete different responses with respect to the final stable fixed state. It is then worth pointing out that the energetic formulation, also extended by (EI), may be unable to characterize the final state after a jump.

\begin{tabular}{|c|c|c|c|}
\hline & & Final state & Dissipated energy \\
\hline $\begin{array}{l}\text { Viscous solution } \\
(\eta \neq 0, m=0)\end{array}$ & - & Closest (Fig. 10) & Friction and viscosity \\
\hline \multirow{2}{*}{$\begin{array}{l}\text { Inertia solution } \\
(\eta=0, m \neq 0)\end{array}$} & $\mu_{0} \leq 3 \mu_{1}$ & Energetic sol. (Fig. 11b) & \multirow{2}{*}{ Only friction } \\
\hline & $\mu_{0} \geq 3 \mu_{1}$ & variable (Figs. 11c, 12a) & \\
\hline \multirow{2}{*}{$\begin{array}{l}\text { Viscous-inertia } \\
\text { tion } \\
(\eta \neq 0, m \neq 0)\end{array}$} & $\xi \geq 1$ & Closest (Fig. 13a) & \multirow{2}{*}{ Friction and viscosity } \\
\hline & $\xi<1$ & Variable (Figs. 12b, 13b) & \\
\hline
\end{tabular}

Table 1: Summary of rate-dependent responses at a jump transition

The role of the kinetic energy in the sledge example is slightly different to the one in the work of Lazzaroni et all. [28] about a dynamic peeling test on a heterogeneous substrate with a small defect with lower toughness. Indeed, in this case, Lazzaroni et al. show the dynamic limit be completely different from the quasi-static energetic description because of the partial, but not vanishing, conversion of some 
potential energy into kinetic energy, acting as an energetic reservoir, by means of shock waves which reduce the energy release rate and slow down the fracture propagation, at least at the first stage. This reservoir role is even more evident in the case of the interruption of the loading process [28, Sec. 2.6] where shock waves continue indefinitely to travel between one end and the not-evolving crack tip of the peeled section. Nevertheless, both the dynamic solution and the energetic solution governed by the here improved energetic formulation predict the same final jump state of the peeled region. Here, instead, the activated kinetic energy at a jump is "immediately" damped by friction and, if any, by viscosity and the dynamic limit behaviour of the sledge strongly depends on the limit value of $\xi$. If we adopt the same time rescaling as in [28], that is, $u(t)=T=\varepsilon t$ with $\varepsilon$ being a very small parameter and $T$ the new evolution parameter, then the scaling laws in (34) for the viscous coefficient and the mass are $\eta^{\varepsilon}=\varepsilon \eta$ and $m^{\varepsilon}=\varepsilon^{2} m$. The parameter $\xi$ then turns out to be invariant with the time-rescaling since $\xi^{\varepsilon}:=\eta^{\varepsilon} /\left(2 \sqrt{m^{\varepsilon}}\right)=\xi$. In general, the final jump does not coincide in this case with the energetic solution regardless the time-rescaling and is extremely sensible to the value of $\xi$ through $m$ and $\eta$.

In addition, one can not exclude a-priori the occurrence of other phenomena during the fast jump transition. For higher degree of freedom systems, the rate-dependent problem, and hence the dissipation and final stable fixed state after a discontinuity, may be strongly affected by the regularization choice on each single variable. For instance, even in two dimensions and limited to the vanishing-viscosity approach, the difference in the choice of the viscous regularization parameters gives rise to a different limiting behaviour, as pointed out in [60, Sec. 2.2]. The same parameter-sensibility behaviour is obviously expected also when inertia is taken into account.

\section{An updated version of the energetic formulation}

Despite the necessity, for achieving an "accurate" solution, to explicitly resolve the rate-dependent problem of a rate-independent system at a discontinuous transition, we don't want to give up to a truly rate-independent formulation which has many unquestionable benefits. Motivated by the previous analyses, an improved energetic formulation, based on extended energetic criteria, is here proposed.

A process $\boldsymbol{z}_{t}: t \rightarrow Z$ is called improved energetic solution if $\forall t \in[0, T]$ it satisfies:

(U1) the local stability condition (st);

(U2) the energy imbalance (EI);

(U3) the extended energy balance

$$
\mathscr{E}\left(\boldsymbol{z}_{t} ;[0, t]\right)+\sum_{t_{j} \in J\left(\boldsymbol{z}_{t}\right)} \mathscr{J}\left(\boldsymbol{z}_{j}^{-}, \boldsymbol{z}_{j}^{+}\right)=0, \quad \forall t \in[0, T]
$$

following from (EI) and $\mathscr{J}$ defined as

$$
\mathscr{J}\left(\boldsymbol{z}_{j}^{-}, \boldsymbol{z}_{j}^{+}\right):=-\mathscr{E}\left(\boldsymbol{z}_{s} ;\left[s_{j}^{-}, s_{j}^{+}\right]\right)
$$

with $s_{j}^{ \pm}=\lim _{\varepsilon \rightarrow 0} \tilde{t}^{-1}\left(t_{j} \pm|\varepsilon|\right)$ and $\boldsymbol{z}_{s}$ linearly interpolated from $\boldsymbol{z}_{j}^{-}$to $\boldsymbol{z}_{j}^{+}$. From (EI) and (56) it follows that

$$
\mathscr{J}\left(\boldsymbol{z}_{j}^{-}, \boldsymbol{z}_{j}^{+}\right) \geq 0, \quad \forall t_{j} \in J\left(\boldsymbol{z}_{t}\right)
$$

(U4) a complementary constitutive energetic criterion at a jump as, for instance,

$$
\boldsymbol{z}_{j}^{+}=\left\{\begin{array}{l}
\underset{\tilde{\boldsymbol{z}} \in \widetilde{\mathcal{S}}}{\arg \max } \mathscr{J}\left(\boldsymbol{z}_{j}^{-}, \tilde{\boldsymbol{z}}\right) \\
\underset{\tilde{\boldsymbol{z}} \in \widetilde{\mathcal{S}}}{\arg \min } \mathscr{J}\left(\boldsymbol{z}_{j}^{-}, \tilde{\boldsymbol{z}}\right) \\
\underset{\tilde{\boldsymbol{z}} \in \widetilde{\mathcal{S}}}{\arg \operatorname{mean}} \mathscr{J}\left(\boldsymbol{z}_{j}^{-}, \tilde{\boldsymbol{z}}\right) \\
\ldots
\end{array}\right.
$$

where $\widetilde{\mathcal{S}}:=\left\{\tilde{\boldsymbol{z}} \in \mathcal{S}:\left(\right.\right.$ EI-jump) is satisfied with $\hat{\boldsymbol{z}}_{s}(0)=\boldsymbol{z}_{j}^{-}$and $\left.\hat{\boldsymbol{z}}_{s}(1)=\tilde{\boldsymbol{z}}\right\}$.

Statements (U1)-(U4) establish an improved version of the energetic formulation. More specifically, condition (U1) requires that any attained state during the absolute continuous part of the solution process is a local minimum for the total energy with respect to the admissible directional neighborhood states and fixed external actions. Condition (U2) states that the energy quantity $\mathscr{E}$, defined in (EI), is a monotone non-increasing function of the time evolution. In particular, the energy is conserved along continuous 
subprocesses while it may decrease at jump points due to the occurrence of additional rate-dependent effects. Condition (U3) restores the energy balance according to the first principle of thermodynamics by considering a complementary energetic term $\mathscr{J}$ which takes into account rate-dependent phenomena occurring at fast jump transitions. To determine the final state at a jump, a further constitutive requirement is needed, represented by condition (U4), which is an extended version of the one proposed by Stefanelli in [60]. A similar condition was before also explored in [52] in the context of thermodynamics of rate-independent plasticity models, where singular dissipation terms were introduced in order to recover energy balance at discontinuous transitions. Condition (59) let the process to maximally dissipate at a jump and may include the BV-solution or the solution obtained by a viscous regularizations. Condition (60), instead, tends to be minimally dissipative at a jump. Hence it is able to select at jumps the energetic solution, if energetically admissible, or the solution with inertia, without other dissipative effects, provided that $\mathscr{J}=0$. Other kind of selection criteria, such as (61), may be preferred since the presence of both viscous and inertia effects may be not able to guarantee $\mathscr{J}=0$, resulting in an oscillating response in the fast transition.

Concerning the sledge example, the capability of such criteria to be able to describe the rate-dependent problems of Tab. 1 is shown in Tab. 2 and Figures 14-16. Specifically, the viscous solution or the viscousinertia solution with $\xi \geq 1$ is retrieved once (59) is chosen as selection criterion. This solution coincides also with the vanishing-viscosity solution, Figures 14-15. On the contrary, condition (60) is able to correctly describe the inertia solution as well as the energetic solution, provided $\mu_{0}<3 \mu_{1}$, Figures 14a-15a. In all other cases, that is inertia solution with $\mu_{0} \geq 3 \mu_{1}$ and viscous-inertia solution with $\xi<1$, none of the complementary constitutive conditions (U4) are always able to predict the correct final state since the response strongly depends on the constitutive parameters, while conditions (U4) do not, Fig. 16. Indeed, Fig. 16a and Fig. 16b show the final fixed stable state rispectively for the inertia solution when $\mu_{0}>3 \mu_{1}$ and for the viscous-inertia solution when $\xi<1$ against the three criteria (59)-(61).

\begin{tabular}{lclc}
\hline & final state & $\begin{array}{c}\text { complementary constitutive } \\
\text { conditions (U4) }\end{array}$ \\
\hline $\begin{array}{l}\text { viscous solution } \\
(\eta \neq 0, m=0)\end{array}$ & - & closest & $(60)$ \\
\hline $\begin{array}{l}\text { inertia solution } \\
(\eta=0, m \neq 0)\end{array}$ & $\mu_{0} \leq 3 \mu_{1}$ & energetic sol. & $(59)$ \\
\cline { 2 - 4 } & $\mu_{0} \geq 3 \mu_{1}$ & variable & - \\
\hline $\begin{array}{l}\text { viscous-inertia } \\
\text { solution } \\
(\eta \neq 0, m \neq 0)\end{array}$ & $\xi \geq 1$ & closest & $(60)$ \\
\hline
\end{tabular}

Table 2: Summary of rate-dependent responses at a jump transition and corresponding complementary constitutive conditions (U4), which are able to capture the same final state with the updated energetic formulation

It is worth noting that the present solution notion is not always able to satisfy the important uppersemicontinuity property, [43], namely the capability of a sequence of solutions, depending on a variation of the data (e.g. initial state, loading path, ...), to converge to the solution of the problem with the imposed limit data. For instance, concerning the sledge example, in case of a loading-unloading path of the form $\ell(t)=\min \left\{t,-t+2 \mu_{0}-2 \varepsilon\right\}$ with $\varepsilon>0$, namely with the turning instant approaching $\mu_{0}^{-}$, the limit solution as $\varepsilon \rightarrow 0$ will not jump while the solution with $\varepsilon=0$ does. Nevertheless, it is possible to recover this property once condition (U1) is replaced by a "stationarity condition", like the (large) first-order stability condition (11), or the differential inclusion of BV-solutions. In this last case, the present updated energetic formulation would differ from BV-solutions only for the selection criteria (U4) which are more general and capable of taking into account the real physics at a jump.

For higher-order problems (discrete or continuous) and where at a jump point infinite candidate solutions exist, the improved energetic formulation still continues to allow the non-uniqueness of the response due both to the occurrence of bifurcation paths or to the existence of multiple states satisfying (U4). In these contexts, conditions like (59)-(61) appear to be extremely weak. A possible improvement of (U4) could be to use a gradient-flow argument for the development of more sophisticated selection criteria. For instance, the final jump state can be first sought on the same direction of the unstable test direction which maximizes the energy loss in the neighborhood of the jump point. A deeper analysis of such criteria 


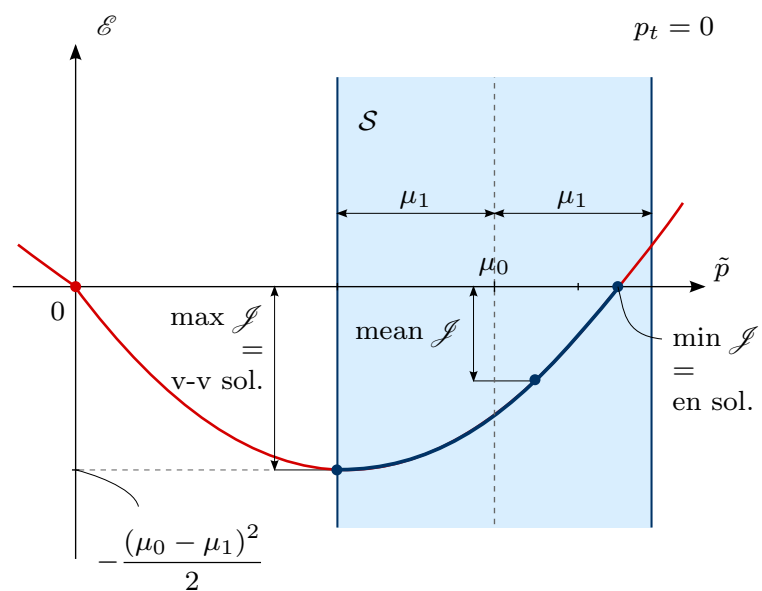

(a) $\mu_{0} \leq 3 \mu_{1}$

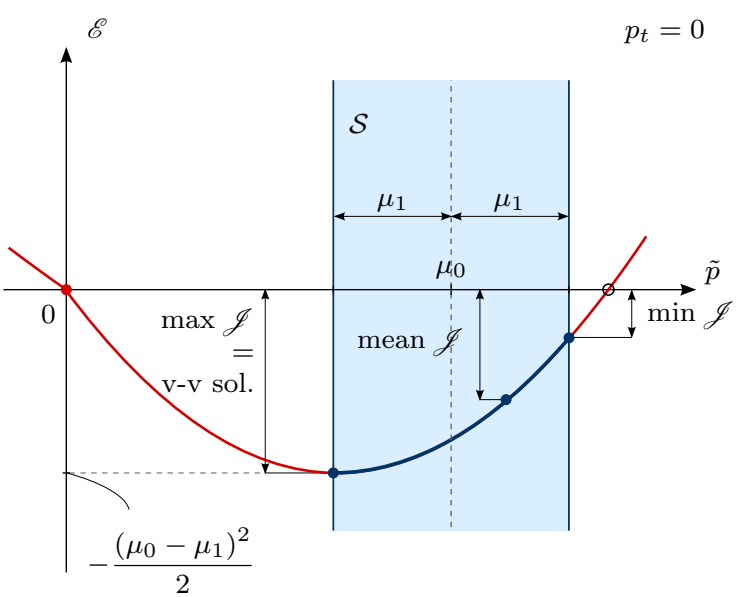

(b) $\mu_{0}>3 \mu_{1}$

Fig. 14: Energy landscape $\mathscr{E}$ at $(t, p)=\left(\mu_{0}, 0\right)$ for the improved energetic formulation for different friction coefficients and comparisons with the vanishing-viscosity solution ( $\mathrm{v}-\mathrm{v}$ sol.) and the local energetic solution (en sol.)

is still in a development stage and will be presented in future works as well as multidimensional examples and comparisons with other solution notions.

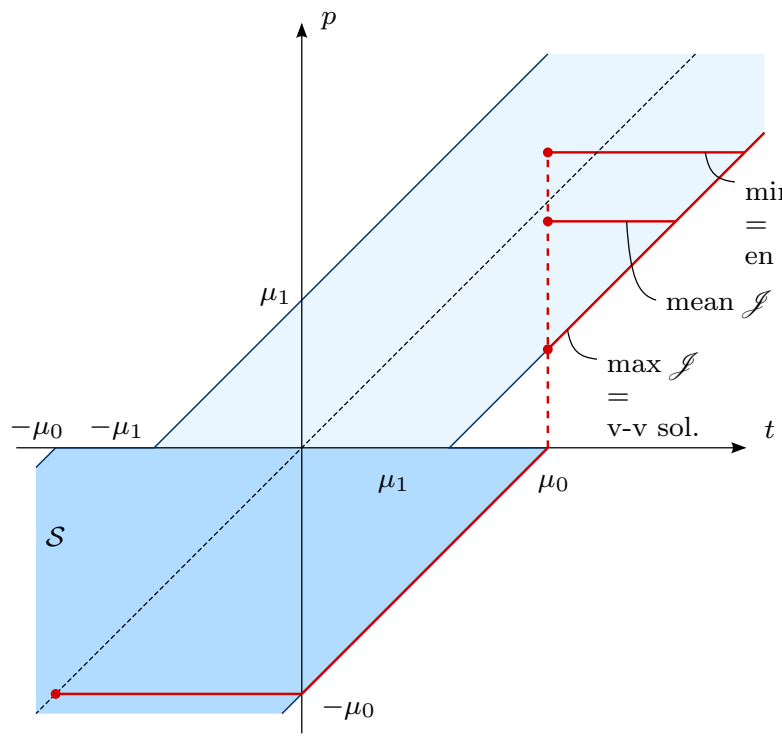

(a) $\mu_{0} \leq 3 \mu_{1}$

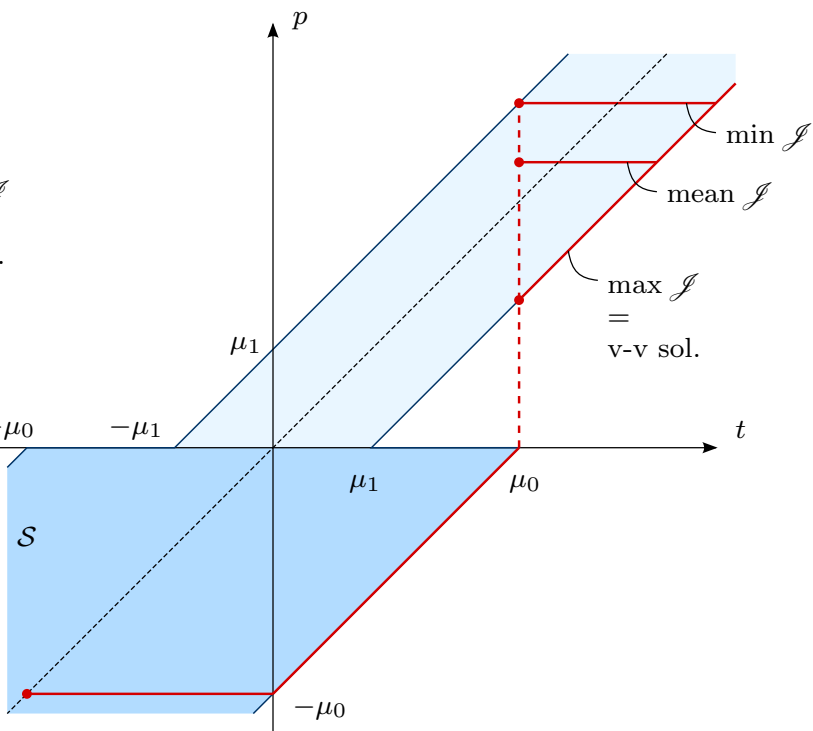

(b) $\mu_{0}>3 \mu_{1}$

Fig. 15: Solution processes for the improved energetic formulation and comparison with the vanishingviscosity solution (v-v sol.) and the local energetic solution (en sol.)

\section{Conclusions}

In this work we have discussed the energetic formulation and highlighted some open issues, such as early jumps and energy conservation at jumps, by means of a simple one-dimensional example. The rate-independent evolution, determined with the energetic formulation, has been compared to the ratedependent response, obtained by introducing both viscous and inertia effects. The discrepancy that arose has been discussed, motivating the step-by-step development of an improved energetic formulation. 


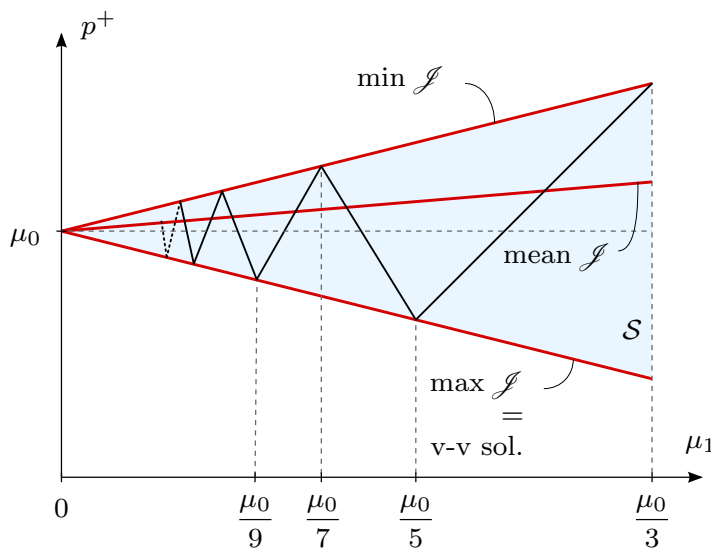

(a)

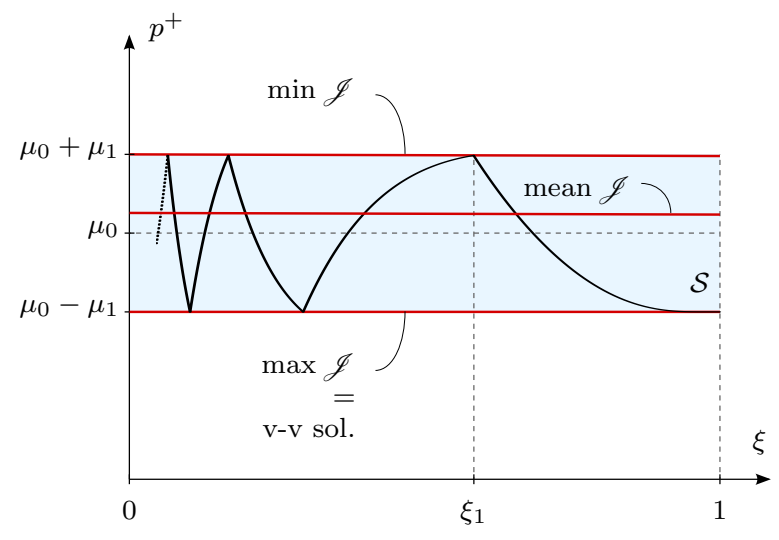

(b)

Fig. 16: Final state $p^{+}$at a jump transition and its dependence respectively on $\mu_{1}$, for the inertia solution when $\mu_{0}>3 \mu_{1}$, (a), and on $\xi$, for the viscous-inertia solution when $\xi<1$, (b). Red lines represent the final state predicted by the complementary constitutive jump criteria (59)-(61) of condition (U4)

In particular, early and nonphysical jumps are avoided taking advantage of a time-reparametrization and an energy-barrier argument which descends by enforcing an energy imbalance during time-discontinuous transitions. The final state after a jump is determined with a new energetic selective criterion which embeds, intrinsically, the energy contribution of rate-dependent phenomena as inertia and viscosity that may arise during fast discontinuous transitions.

An improved version of the energetic formulation is finally proposed, preserving completely the rateindependent setting.

Acknowledgements The author acknowledges the financial support of INdAM-GNFM through the program: "Progetto Giovani". The author is immensely grateful to Davide Bernardini, Giuliano Lazzaroni, Adriana Garroni and Matteo Negri for fruitful and crucial discussions, key suggestions and for providing relevant references. The author thanks also Achille Paolone for the transmitted enthusiasm for this work and the first Reviewer for the precise and appropriate remarks.

\section{References}

1. Agostiniani, V.: Second order approximations of quasistatic evolution problems in finite dimension. Discrete and Continuous Dynamical Systems - Series A 32(4), 1125-1167 (2012)

2. Alessi, R., Bernardini, D.: Analysis of localization phenomena in Shape Memory Alloys bars by a variational approach. International Journal of Solids and Structures 73-74, 113-133 (2015)

3. Alessi, R., Marigo, J.J., Vidoli, S.: Gradient Damage Models Coupled with Plasticity and Nucleation of Cohesive Cracks. Archive for Rational Mechanics and Analysis 214(2), 575-615 (2014)

4. Alessi, R., Marigo, J.J., Vidoli, S.: Gradient damage models coupled with plasticity: Variational formulation and main properties. Mechanics of Materials 80, Part B(0), 351-367 (2015)

5. Alessi, R., Pham, K.: Variational formulation and stability analysis of a three dimensional superelastic model for shape memory alloys. Journal of the Mechanics and Physics of Solids 87, 150-176 (2016)

6. Ambati, M., De Lorenzis, L.: Phase-field modeling of brittle and ductile fracture in shells with isogeometric NURBS-based solid-shell elements. Computer Methods in Applied Mechanics and Engineering, In Press (2016)

7. Ambati, M., Kruse, R., De Lorenzis, L.: A phase-field model for ductile fracture at finite strains and its experimental verification. Computational Mechanics 57(1), 149-167 (2015)

8. Amor, H., Marigo, J.J., Maurini, C.: Regularized formulation of the variational brittle fracture with unilateral contact: Numerical experiments. Journal of the Mechanics and Physics of Solids 57(8), 1209-1229 (2009)

9. Auricchio, F., Mielke, A., Stefanelli, U.: A rate-independent model for the isothermal quasi-static evolution of shape-memory materials. Mathematical Models and Methods in Applied Sciences 18(125), 125-164 (2008)

10. Bažant, Z.P., Cedolin, L.: Stability of Structures: Elastic, Inelastic, Fracture, and Damage Theories (2003) 
11. Bourdin, B., Francfort, G.A., Marigo, J.J.: Numerical experiments in revisited brittle fracture. Journal of the Mechanics and Physics of Solids 48(4), 797-826 (2000)

12. Bourdin, B., Francfort, G.A., Marigo, J.J.: The Variational Approach to Fracture. Journal of Elasticity 91(1-3), 5-148 (2008)

13. Cagnetti, F.: A vanishing viscosity approach to fracture growth in a cohesive zone model with prescribed crack path. Mathematical Models and Methods in Applied Sciences 18(07), 1027-1071 (2011)

14. Chambolle, A., Giacomini, A., Ponsiglione, M.: Crack Initiation in Brittle Materials. Archive for Rational Mechanics and Analysis 188(2), 309-349 (2007)

15. Crismale, V.: Globally stable quasistatic evolution for a coupled elastoplasticdamage model. ESAIM: Control, Optimisation and Calculus of Variations, Accepted Paper (2015)

16. Crismale, V., Lazzaroni, G.: Viscous approximation of quasistatic evolutions for a coupled elastoplastic-damage model. Preprint SISSA 05/2015/MATE (2015)

17. Dal Maso, G., De Simone, A., Mora, M.G.: Quasistatic Evolution Problems for Linearly Elasticity Perfectly Plastic Materials. Archive for Rational Mechanics and Analysis 180(2), 237-291 (2006)

18. Dal Maso, G., De Simone, A., Mora, M.G., Morini, M.: Globally stable quasistatic evolution in plasticity with softening. Netw. Heterog. Media 3, 567-614 (2008)

19. Dal Maso, G., Orlando, G., Toader, R.: Fracture models for elasto-plastic materials as limits of gradient damage models coupled with plasticity: the antiplane case. Preprint SISSA 04/2015/MATE (2015)

20. Efendiev, M.A., Mielke, A.: On the Rate-Independent Limit of Systems with Dry Friction and Small Viscosity 13(1), 151-167 (2006)

21. Francfort, G.A., Garroni, A.: A Variational View of Partial Brittle Damage Evolution. Archive for Rational Mechanics and Analysis 182(1), 125-152 (2006)

22. Francfort, G.A., Giacomini, A., Marigo, J.J.: The taming of plastic slips in von mises elasto-plasticity. Submitted Paper 2014

23. Freddi, F., Royer-Carfagni, G.: Regularized variational theories of fracture: A unified approach. Journal of the Mechanics and Physics of Solids 58(8), 1154-1174 (2010)

24. Halphen, B., Nguyen, Q.S.: Generalized Standard Materials. Journal de Mécanique 14(1), 39-63 (1975)

25. Lancioni, G.: Modeling the Response of Tensile Steel Bars by Means of Incremental Energy Minimization. Journal of Elasticity (2015)

26. Larsen, C.J.: Epsilon-stable quasi-static brittle fracture evolution. Communications on Pure and Applied Mathematics pp. NA-NA (2009)

27. Larsen, C.J.: Local minimality and crack prediction in quasi-static Griffith fracture evolution. Discrete and Continuous Dynamical Systems - Series S 6(1), 121-129 (2013)

28. Lazzaroni, G., Bargellini, R., Dumouchel, P.E., Marigo, J.J.: On the role of kinetic energy during unstable propagation in a heterogeneous peeling test. International Journal of Fracture 175(2), 127-150 (2012)

29. Lazzaroni, G., Rossi, R., Thomas, M., Toader, R.: Rate-independent damage in thermo-viscoelastic materials with inertia. Preprint SISSA 52/2014/MATE (2014)

30. Lazzaroni, G., Toader, R.: A model for crack propagation based on viscous approximation. Mathematical Models and Methods in Applied Sciences 21(10), 2019-2047 (2011)

31. Lazzaroni, G., Toader, R.: Energy release rate and stress intensity factor in antiplane elasticity. Journal de Mathématiques Pures et Appliquées 95(6), 565-584 (2011)

32. Lazzaroni, G., Toader, R.: Some remarks on the viscous approximation of crack growth. Discrete and Continuous Dynamical Systems - Series S 6(1), 131-146 (2013)

33. León Baldelli, A.A., Babadjian, J.F., Bourdin, B., Henao, D., Maurini, C.: A variational model for fracture and debonding of thin films under in-plane loadings. Journal of the Mechanics and Physics of Solids 70, 320-348 (2014)

34. León Baldelli, A.A., Maurini, C., Pham, K.: A gradient approach for the macroscopic modeling of superelasticity in softening shape memory alloys. International Journal of Solids and Structures 52, 45-55 (2015)

35. Marigo, J.J.: Initiation of Cracks in Griffith's Theory: An Argument of Continuity in Favor of Global Minimization. Journal of Nonlinear Science 20(6), 831-868 (2010)

36. Mesgarnejad, A., Bourdin, B., Khonsari, M.: Validation simulations for the variational approach to fracture. Computer Methods in Applied Mechanics and Engineering 290, 420-437 (2015)

37. Mielke, A.: A Mathematical Framework for Generalized Standard Materials in the Rate-Independent Case. In: R. Helmig, A. Mielke, B. Wohlmuth (eds.) Multifield Problems in Solid and Fluid Mechanics, Lecture Notes in Applied and Computational Mechanics, vol. 28, pp. 399-428. Springer Berlin / Heidelberg (2006) 
38. Mielke, A.: Lipschitz Lecture held in Bonn: Modeling and analysis of rate-independent processes. Tech. rep. (2007)

39. Mielke, A.: Differential, Energetic, and Metric Formulations for Rate-Independent Processes, pp. 87-169. Springer Lecture Notes in Mathematics (2011)

40. Mielke, A., Rossi, R., Savaré, G.: Modeling solutions with jumps for rate-independent systems on metric spaces. Discrete and Continuous Dynamical Systems 25(2), 585-615 (2009)

41. Mielke, A., Rossi, R., Savaré, G.: BV solutions and viscosity approximations of rate-independent systems. ESAIM: Control, Optimisation and Calculus of Variations 18(01), 36-80 (2012)

42. Mielke, A., Rossi, R., Savaré, G.: Balanced Viscosity (BV) solutions to infinite-dimensional rateindependent systems. Journal of the European Mathematical Society To appear (2014)

43. Mielke, A., Roubíček, T.: Rate-Independent Systems: Theory and Application. Springer (2015)

44. Mielke, A., Theil, F.: On rate-independent hysteresis models. NoDEA : Nonlinear Differential Equations and Applications 11(2), 151-189 (2004)

45. Negri, M.: Quasi-static rate-independent evolutions: characterization, existence, approximation and application to fracture mechanics. ESAIM Control Optim. Calc. Var. 20(4), 983-1008 (2014)

46. Negri, M.: Quasi-static evolutions in brittle fracture generated by gradient flows: sharp crack and phase-field approaches. preprint (2015)

47. Nguyen, Q.S.: Standard dissipative systems and stability analysis. John Wiley \& Sons (2000)

48. Ortiz, M., Repetto, E.A.: Nonconvex energy minimization and dislocation structures in ductile single crystals. Journal of the Mechanics and Physics of Solids 47(2), 397-462 (1999)

49. Pham, K., Marigo, J.J.: From the onset of damage to rupture: construction of responses with damage localization for a general class of gradient damage models. Continuum Mechanics and Thermodynamics 25(2-4), 147-171 (2011)

50. Pham, K., Marigo, J.J.: Stability of Homogeneous States with Gradient Damage Models: Size Effects and Shape Effects in the Three-Dimensional Setting. Journal of Elasticity 110(1), 63-93 (2012)

51. Pham, K., Marigo, J.J., Maurini, C.: The issues of the uniqueness and the stability of the homogeneous response in uniaxial tests with gradient damage models. Journal of the Mechanics and Physics of Solids 59(6), 1163-1190 (2011)

52. Puglisi, G., Truskinovsky, L.: Thermodynamics of rate-independent plasticity. Journal of Mechanics Physics of Solids 53, 655-679 (2005)

53. Rossi, R., Savaré, G.: A characterization of energetic and $\$ B V \$$ solutions to one-dimensional rateindependent systems. Discrete and Continuous Dynamical Systems - Series S 6(1), 167-191 (2013)

54. Rossi, R., Thomas, M.: Coupling rate-independent and rate-dependent processes: existence results. Preprint CVGMT pp. 1-51 (2015)

55. Roubíček, T.: Rate-independent processes in viscous solids at small strains. Mathematical Methods in the Applied Sciences 32(7), 825-862 (2009)

56. Roubíček, T.: Thermodynamics of Rate-independent Processes in Viscous Solids at Small Strains. SIAM Journal on Mathematical Analysis 42(1), 256-297 (2010)

57. Roubíček, T.: Maximally-dissipative local solutions to rate-independent systems and application to damage and delamination problems. Nonlinear Analysis: Theory, Methods \& Applications 113, 33-50 (2015)

58. Roubíček, T., Panagiotopoulos, C.G., Mantič, V.: Local-solution approach to quasistatic rateindependent mixed-mode delamination. Mathematical Models and Methods in Applied Sciences 25(07), 1337-1364 (2015)

59. Solombrino, F.: Quasistatic Evolution in Perfect Plasticity for General Heterogeneous Materials, vol. $212(2013)$

60. Stefanelli, U.: A variational characterization of rate-independent evolution. Mathematische Nachrichten 282(11), 1492-1512 (2009)

61. Worden, K., Tomlinson, G.: Nonlinearity in Structural Dynamics: Detection, Identification and Modelling (2000) 